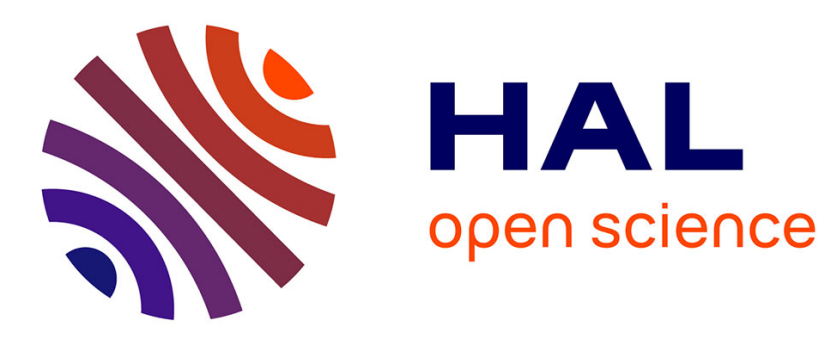

\title{
Named Data Networking in Vehicular Ad hoc Networks: State-of-the-Art and Challenges
}

Hakima Khelifi, Senlin Luo, Boubakr Nour, Hassine Moungla, Yasir Faheem, Rasheed Hussain, Adlen Ksentini

\section{- To cite this version:}

Hakima Khelifi, Senlin Luo, Boubakr Nour, Hassine Moungla, Yasir Faheem, et al.. Named Data Networking in Vehicular Ad hoc Networks: State-of-the-Art and Challenges. Communications Surveys and Tutorials, IEEE Communications Society, 2019, pp.1-1. 10.1109/COMST.2019.2894816 . hal02100996

\section{HAL Id: hal-02100996 \\ https://hal.science/hal-02100996}

Submitted on 16 Apr 2019

HAL is a multi-disciplinary open access archive for the deposit and dissemination of scientific research documents, whether they are published or not. The documents may come from teaching and research institutions in France or abroad, or from public or private research centers.
L'archive ouverte pluridisciplinaire HAL, est destinée au dépôt et à la diffusion de documents scientifiques de niveau recherche, publiés ou non, émanant des établissements d'enseignement et de recherche français ou étrangers, des laboratoires publics ou privés. 


\title{
Named Data Networking in Vehicular Ad hoc Networks: State-of-the-Art and Challenges
}

\author{
Hakima Khelifi, Senlin Luo, Boubakr Nour, Hassine Moungla, Yasir Faheem, Rasheed Hussain, \\ and Adlen Ksentini
}

\begin{abstract}
Information-Centric Networking (ICN) has been proposed as one of the future Internet architectures. It is poised to address the challenges faced by today's Internet that include, but not limited to, scalability, addressing, security, and privacy. Furthermore, it also aims at meeting the requirements for new emerging Internet applications. To realize ICN, Named Data Networking (NDN) is one of the recent implementations of ICN that provides a suitable communication approach due to its clean slate design and simple communication model. There are a plethora of applications realized through ICN in different domains where data is the focal point of communication. One such domain is Intelligent Transportation System (ITS) realized through Vehicular Ad hoc NETwork (VANET) where vehicles exchange information and content with each other and with the infrastructure. To date, excellent research results have been yielded in the VANET domain aiming at safe, reliable, and infotainment-rich driving experience. However, due to the dynamic topologies, host-centric model, and ephemeral nature of vehicular communication, various challenges are faced by VANET that hinder the realization of successful vehicular networks and adversely affect the data dissemination, content delivery, and user experiences. To fill these gaps, NDN has been extensively used as underlying communication paradigm for VANET.

Inspired by the extensive research results in NDN-based VANET, in this paper, we provide a detailed and systematic review of NDN-driven VANET. More precisely, we investigate the role of NDN in VANET and discuss the feasibility of NDN architecture in VANET environment. Subsequently, we cover in detail, NDN-based naming, routing and forwarding, caching, mobility, and security mechanism for VANET. Furthermore, we discuss the existing standards, solutions, and simulation tools used in NDN-based VANET. Finally, we also identify open challenges and issues faced by NDN-driven VANET and highlight future research directions that should be addressed by the research community.
\end{abstract}

Index Terms-Information-Centric Networking, Named Data Networking, Vehicular Ad-hoc Networks, Survey

\section{INTRODUCTION}

D uring the last couple of decades, Vehicular Ad-hoc Networks (VANETs) [1] gained a lot of attention from industry and academia to improve driving safety, efficiently

The work of S. Luo was supported by the National 242 Project under Grant No. 2017A149 (Corresponding author: Senlin Luo).

H, Khelifi, S. Luo, and B. Nour are with Beijing Institute of Technology, China (emails: hakima@bit.edu.cn, luosenlin@bit.edu.cn, n.boubakr@bit.edu.cn).

H. Moungla is with University of Paris Descartes and Institute Mines Telecom, France (email: hassine.moungla@parisdescartes.fr).

Y. Faheem is with COMSATS University Islamabad, Islamabad campus, Pakistan (email: yasir.faheem@comsats.edu.pk).

R. Hussain is with Innopolis University, Innopolis, Russia (email: r.hussain@innopolis.ru ).

A. Ksentini is with Eurecom, France (email: adlen.ksentini@eurecom.fr). manage traffic conditions, and enhance user experience during driving. To achieve these goals, several protocols, solutions, and applications such as Dedicated Short Range Communications (DSRC) [2] and Wireless in Vehicular Environments (WAVE), have been proposed. However, due to its inherited features from parental Mobile Ad hoc NETwork (MANET) such as ephemerality, high mobility, and intermittency, the design of network and communication protocols for VANETs is challenging. The use of IP protocol to improve content delivery, vehicular mobility management, consistent routing, and topology management makes VANETs more complex to cope with application requirements and quality of service (QoS) provisions. Thus, it is imperative to think of another communication architecture that befits the communication requirements of the VANET applications and services [3].

To this end, the current Internet architecture does not gracefully scale with the increasing as well as complex requirements of the diversified applications. In essence, the Internet was designed from entity-oriented standpoint where it provides a communication platform for entities through established "connection". However, over the decades, the Internet became business-oriented and content-oriented, representing a platform to share content regardless of the source of the content. This, at least in part, happened due to the proliferation of content-oriented applications over the years. These content-oriented applications cover different domains such as entertainment, news, sports, business, health, education, press, shopping and so forth. The content-centric nature of these applications, on one hand provides value and utility to users, but on the other hand causes disparity among the current Internet architecture. This phenomenon advocates for the design of new Internet architecture that prioritizes the content itself instead of the source of the content. Another important perspective is that future Internet gracefully supports the idea of content-driven Internet rather than connectiondriven Internet.

Recently, National Research Foundation (NSF) [4] funded different projects targeting the Future Internet Architecture including Named-Data Network (NDN) [5], Mobility First [6], Nebula [7], eXpressive Internet Architecture (XIA) [8], and ChoiceNet [9]. On the other hand, UC Berkeley and European Commission (EU) [10] also funded a variety of projects as a new vision for the future Internet including DataOriented Network Architecture (DONA) [11], Content Mediator Architecture for Content-Aware Networks (COMET) [12], Convergence [13], Scalable and Adaptive Internet Solutions (SAIL) [14], and Publish-Subscribe Internet Technology (PURSUIT) [15]. Among other potential candidates for 
TABLE I

LIST OF ACRONYMS USED

\begin{tabular}{|l|l|l|l|}
\hline Acronyms & \multicolumn{1}{|c|}{ Definitions } & Acronyms & \multicolumn{1}{c|}{ Definitions } \\
\hline$C C N$ & Content Centric Networking & $Q o S$ & Quality of Service \\
\hline ccnSim & Content Centric Networking Simulator & $R S U$ & Road Side Units \\
\hline$C S$ & Content Store & $S D N$ & Software Defined Networking \\
\hline$F I B$ & Forwarding Information Base & $T C P / I P$ & Transmission Control Protocol/Internet Protocol \\
\hline$I C N$ & Information-Centric Networking & $V 2 I$ & Vehicle to remote infrastructure \\
\hline$I o T$ & Internet of Things & $V 2 R$ & Vehicle to Roadside Units \\
\hline$I T S$ & Intelligent Transportation System & $V 2 U$ & Vehicle to Uniform \\
\hline$N D N$ & Named Data Networking & $V 2 V$ & Vehicle-to-Vehicle \\
\hline$n d n S I M$ & Named Data Networking Simulator & $V 2 X$ & Vehicle to everything \\
\hline$N S 2 / 3$ & Network Simulator 2/3 & $V A N E T s$ & Vehicular Ad-hoc Networks \\
\hline$P I T$ & Pending Interest Table & $V C C N$ & Vehicular Content Centric Networking \\
\hline$Q o E$ & Quality of Experience & $V N D N$ & Vehicular Name Data Networking \\
\hline
\end{tabular}

future Internet architecture, Information-Centric Networking (ICN) [16] has been proposed as a new networking paradigm for the future Internet. ICN addresses the pressing issues faced by the current IP-based networks [17], aiming to ensure efficient data dissemination by integrating all network features around the name of content rather than the host address. In ICN, the content name is the fundamental element (also considered as first-class citizen of the network), where it should be globally unique, persistent, secure, location-independent, and friendly. Content names can be represented in different formats such as hierarchical, flat, or attribute-value names. In this context, the traditional routing plane of the IP-based networks has been changed from using host address to using content name, known as name-based routing. Moreover, in-network caching and replication are used during the communication to allow fast data retrieval.

To realize the vision of ICN, different solutions have been developed using the ICN communication paradigm. Named Data Networking, which is an implementation of ICN that has been forked from Content-Centric Networking (CCN) [18], was the only full-scale realization of the future Internet architecture. NDN focuses on content name as the key element in communication rather than IP address where each content in the network is assigned a unique and persistent name. Consumers request content based on its name and the intermediate nodes use the requested name to deliver it to the content provider or replica-nodes. Further, each content is decoupled from its provider's location and security mechanisms are applied to the content instead of securing the communication channel. Hence, intermediate-nodes can cache the received content to re-use it in future requests rather than forwarding the request to the original provider. This improves the network performance and content delivery. Therefore, the straight-forward, simple, and efficient design of NDN (the implementation already referred to as Vanilla NDN) makes it a perfect candidate for content-centric networks [5].

Apart from the afore-mentioned reasons, the paradigm shift from the current TCP/IP-based networking is also necessary due to the issues that current Internet architecture is facing, for instance, routing, transport, congestion, scalability, security, and so on. Whilst routing in IP networks suffers from link failures, stability of the routing path, and look-up process, broadcast and multicast are expensive from both computation and communication perspective and incur additional overhead for the network, while TCP-style reliable communication is often not suitable for QoS control and data prioritization. On the other hand, security is an after-thought in the current Internet; therefore, adding security mechanisms to the current Internet degrades the performance.

Inspired from the features of NDN, the communication architecture of VANET has been modified to natively use NDN [19], [20]. To date, researchers have proposed integrated solutions for VANET with NDN, with clean application design, customized naming and caching schemes, optimized forwarding strategies, and seamless mobility support. However, this integration introduces several issues and challenges. In this paper, we take a holistic approach towards NDN-based VANET and carry out a comprehensive overview of VANET with the new communication paradigm. Table I lists all the acronyms and their explanations used in this paper.

\section{A. Related Surveys}

As stated earlier, the content-oriented nature of VANET advocates for a communication architecture other than IPbased architecture. To this end, ICN is one of the best candidates for VANET to serve as communication architecture. The research on NDN-driven VANET is still in its infancy and it is expected that NDN will play a pivotal role in the commercial realization of connected car technology. Existing literature focuses on solutions for NDN-driven VANET from the perspective of efficient, stable, and reliable communication. These solutions mainly address the integration of VANET with ICN (more precisely NDN) [20], [26], where a handful of researches and surveys are available in the literature that 
TABLE II

COMPARISON OF CONTRIBUTION OF RELATEd SURVEy PAPERS

\begin{tabular}{|c|c|c|c|c|c|c|c|c|c|c|c|c|c|}
\hline 离 & ¿্స & 量 & 哭 & 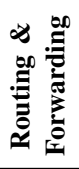 & 己ֶ. & 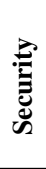 & 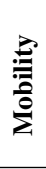 & 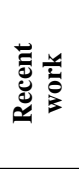 & 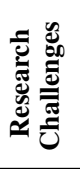 & 窇 & 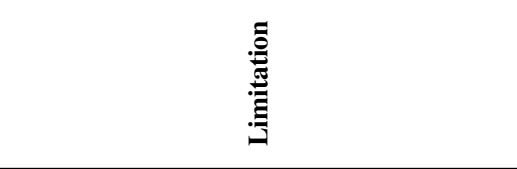 & 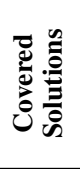 & 己ें \\
\hline [21] & 2015 & $x$ & $\checkmark$ & $\checkmark$ & $\checkmark$ & $\checkmark$ & $x$ & $x$ & $\checkmark$ & $x$ & $\begin{array}{l}\text { - Not comprehensive and missing recent } \\
\text { relevant literature. } \\
\text { - Mobility is not covered. }\end{array}$ & 9 & [2010-2014] \\
\hline$[22]$ & 2016 & $\checkmark$ & $\checkmark$ & $\checkmark$ & $\checkmark$ & $x$ & $x$ & $x$ & $x$ & $\checkmark$ & $\begin{array}{l}\text { - Missing recent relevant literatures. } \\
\text { - Mobility and Security aspects not covered. }\end{array}$ & 22 & [2012-2016] \\
\hline [20] & 2016 & $x$ & $\checkmark$ & $\checkmark$ & $\checkmark$ & $\checkmark$ & $\checkmark$ & $x$ & $x$ & $\checkmark$ & $\begin{array}{l}\text { - Concept paper and missing details about } \\
\text { recent relevant literatures. }\end{array}$ & 11 & [2010-2014] \\
\hline [23] & 2016 & $x$ & $x$ & $x$ & $x$ & $\checkmark$ & $x$ & $x$ & $\checkmark$ & $x$ & $\begin{array}{l}\text { - Lack thoroughness and comprehensive- } \\
\text { ness, no details on the topic. } \\
\text { - Discuss only security, misses naming, } \\
\text { caching, mobility, routing and forwarding } \\
\text { aspects. }\end{array}$ & 5 & [2012-2015] \\
\hline [24] & 2016 & $x$ & $x$ & $\checkmark$ & $x$ & $x$ & $x$ & $x$ & $\checkmark$ & $x$ & $\begin{array}{l}\text { - Focuses on only one aspect of NDN-based } \\
\text { VANET. } \\
\text { - Misses major aspects such as naming, } \\
\text { caching, mobility and Security. }\end{array}$ & 11 & [2013-2015] \\
\hline [25] & 2017 & $x$ & $x$ & $\checkmark$ & $\checkmark$ & $\checkmark$ & $\checkmark$ & $x$ & $\checkmark$ & $x$ & $\begin{array}{l}\text { - Did not cover the topic comprehensively } \\
\text { and just touched upon NDN architecture in } \\
\text { VANET from bird's view. } \\
\text { - Lacks recent literature and aspects of nam- } \\
\text { ing and mobility. }\end{array}$ & 16 & [2011-2017] \\
\hline [19] & 2017 & $x$ & $\checkmark$ & $\checkmark$ & $\checkmark$ & $\checkmark$ & $x$ & $x$ & $x$ & $\checkmark$ & $\begin{array}{l}\text { - Focuses on ITS and NDN but from a smart } \\
\text { city perspective. } \\
\text { - A concept paper missing the thoroughness } \\
\text { of NDN-based VANET. }\end{array}$ & 5 & [2012-2016] \\
\hline [26] & 2017 & $x$ & $\checkmark$ & $\checkmark$ & $\checkmark$ & $\checkmark$ & $x$ & $x$ & $\checkmark$ & $x$ & $\begin{array}{l}\text { - Do not focus on the traditional VANET } \\
\text { and its challenges. } \\
\text { - Does not cover current state of the art and } \\
\text { research challenges for VANET. }\end{array}$ & 5 & [2015-2017] \\
\hline Our & 2018 & $\checkmark$ & $\checkmark$ & $\checkmark$ & $\checkmark$ & $\checkmark$ & $\checkmark$ & $\checkmark$ & $\checkmark$ & $\checkmark$ & & 101 & [2010-2018] \\
\hline
\end{tabular}

focus on merging NDN with traditional VANETs [19]-[26]. However, the current literature does not cover all major aspects of NDN-based communication in VANETs. We, on the other hand, carry out a comprehensive and systematic survey of NDN-based communication in the context of VANETs. We cover NDN-based VANET communication in a holistic way from the perspective of the state-of-the-art, issues, and future challenges. To justify the need and contribution of this survey, we summarize the existing surveys in Table II, and the details are as follows:

Bouk et al. [21] carried out a brief survey on the feasibility of applying CCN paradigm to Vehicular Communications, referred to as Vehicular CCN (VCCN). They discussed content naming, routing, caching, and security challenges in VCCN. Similarly, Zhu et al. [22] investigated main issues and future research directions in connected vehicles through ICN while focusing on naming, caching components, and routing. Similarly, Amadeo et al. [20] briefly discussed the applicability of ICN in vehicular environment by studying different ICN aspects such as naming, routing, caching, and security from VANET perspective. The authors also identified the main challenges and presented future research directions. Signorello et al. [23] discussed security risks associated with the integration of NDN and VANET, and classified attacks from NDN perspective such as interest flooding attacks, cache poisoning attacks, and name privacy. Whereas Yaqub et al. [24] focused primarily on NDN forwarding plane. The authors provided an overview of the existing Vehicular-ICN (VICN) forwarding schemes, and highlighted the key challenges caused by the interest forwarding strategies in VICNs. In addition, Shemsi et al. [25] conducted a short survey on the existing research progress in NDN-based VANET along with the comparison with traditional TCP/IP architecture. Furthermore, the authors also discussed various research challenges associated with naming, forwarding, mobility, and interest flooding attacks in VANET. Bouk et al. [19] discussed the integration of ITS with NDN by abstractly highlighting the current and future research directions from naming, routing, caching, and QoS standpoint. The authors also presented an abstract NDN architecture for ITS in smart cities. Lastly, Ahmed et al. [26] focused on the applicability of NDN in Software-Defined Vehicular Networks to realize robust and efficient vehicular networks.

From the aforementioned discussion and Table II, it is worth noting that the existing surveys either partially cover 
different aspects of NDN-based VANETs [19]-[22], [25], [26], or focus on a specific aspect [23], [24]. Furthermore, the research carried out in [21], [22], [25] do not cover the entire spectrum of existing and recent solutions in NDN-based VANET. Whereas some exiting works [19], [20], [26] shortly review the existing solutions along with open research issues and future directions without covering the details of the state of the art comprehensively. Table II also provides a comparative summary of the existing surveys and how these surveys differ from our survey including their limitations, covered solutions, and their respective year interval.

To the best of our knowledge, this paper is the first comprehensive effort to review the NDN-based VANET in a holistic way, covering the entire spectrum of the integration. Our contribution can be viewed as a critical and systematic overview of all aspects of NDN-based vehicular communications along with state of the art solutions, issues, and future challenges.

\section{B. Our Contributions}

The existing works as outlined in the previous subsection lack in both depth and breadth of the NDN-based VANET. Current works either focus on particular aspects, or the reviews are outdated. To fill the gaps, we investigate the NDN-based VANET comprehensively by covering state of the art and all other aspects that are of prime importance to the successful realization of NDN-based VANET. We focus on different aspects of the Vehicular NDN including existing architectures, content naming schemes, routing, forwarding strategies, content discovery, in-network caching, mobility solutions, security and privacy, data dissemination, and quality of services. Furthermore, we also discuss the existing standards, solutions, and simulation tools as well as issues and open research challenges with open-ended discussion on the successful proliferation of NDN-based VANETs. The contributions of this paper are summarized below:

- We comprehensively survey NDN-based VANET, covering naming schemes, their classification, and comparative analysis.

- We describe in detail, the current state of the art results on NDN-based VANET from applications and services perspective covering data discovery, forwarding mechanisms, data delivery, in-network caching, mobility, standards and solutions, and simulation techniques in NDNbased VANETs.

- We present an in-depth overview of the issues including security and privacy that hinder the successful realization of the NDN-based VANET.

- We describe the challenges faced by the NDN-based VANET and future research directions.

\section{Organization}

The rest of the paper is organized as follows: Section II discusses brief characteristics, properties, and applications of VANETs. In Section III, we present NDN architecture followed by Section IV, which outlines the integration of NDN and VANET, while Section V discusses the existing NDN-based VANET architecture. In the following sections,

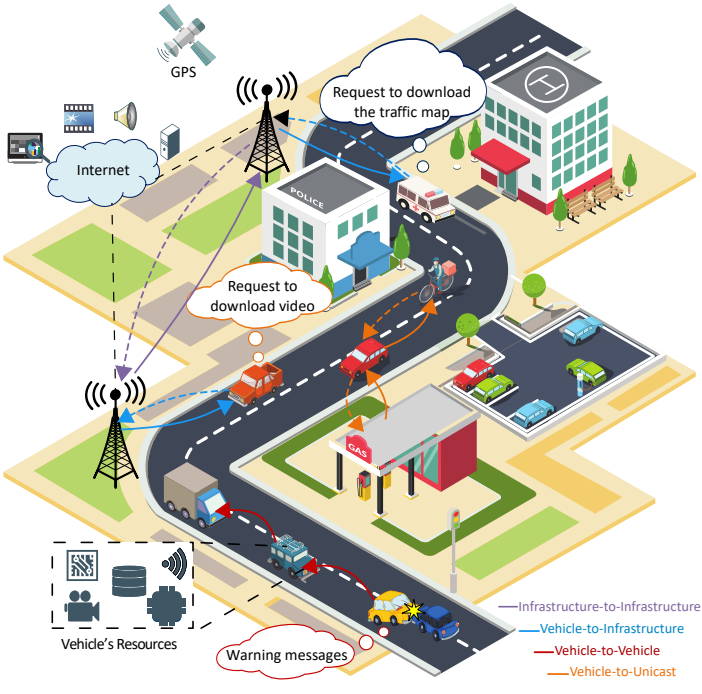

Fig. 1. VANET communication models

we discuss the current state of the art for NDN-based VANET. Particularly, Section VI discusses the naming solutions, and Section VII presents the routing and forwarding strategies, while Section VIII presents a review on in-network caching schemes in NDN-based VANET. We present the mobility solutions in section IX, and security and privacy in Section X. Furthermore, Section XI presents an overview of the existing standards, solutions, and simulation tools for NDN-based VANET. Then, we focus on the issues and challenges for NDN-based VANET in Section XII, and finally we conclude the paper in Section XIII.

\section{An Overview of Vehicular Ad-HOC Network}

Vehicular Ad-hoc Network (VANET) [27] is a special breed of MANET in which vehicles are employed as mobile nodes restricted to road topologies. In the following, we provide an overview of VANETs, including their properties, challenges, and applications.

Due to high speed mobility, diverse mobility patterns, short inter-connection times, and potentially different network architectures, the protocols designed for MANETs do not perform well in VANETs. Therefore, specialized protocols have been designed for VANET. To this end, DSRC is one of the technologies through which vehicular communications are realized [28]. Meanwhile, IEEE has amended the 802.11 standard to be used as WAVE, also referred to as IEEE $802.11 \mathrm{p}$ [29]. 802.11p is an enhanced version of 802.11a. However, both standards are based on Orthogonal Frequency-Division Multiplexing (OFDM). The main difference between them is that $802.11 \mathrm{p}$ has been designed to use $10 \mathrm{MHz}$ frequency bandwidth which is the half of bandwidth that is used by 802.11a. This will make the signal faster, and more robust especially in multipath propagation that is the most important characteristic in vehicular environments.

In VANETs, communication may be classified as Vehicleto-Vehicle (V2V), Vehicle to Road Side Units (V2R), Vehicle to remote Infrastructure (V2I), and Vehicle-to-cloud [30]-[32]. 
Additionally, vehicles ${ }^{1}$ may also communicate with charging stations, personal communication devices, and smart grids, and other location-based servers [33]-[37]. Figure 1 illustrates different communication scenarios in VANET, including communication in-vehicle (e.g., on-board unit, Global Positioning System (GPS), etc.), between vehicles to exchange information, with road-side units and pedestrian, as well as with Internet and could-based services. In VANETs, the data exchanged among different entities usually belongs to two classes of applications, safety- and infotainment-related applications. This data is used for traffic management, safety, value-added services, driver assistance, and information. These applications exhibit different behavior, properties, characteristics, and have different requirements. However, most of these applications rely on the content exchanged among vehicles and external entities [38]. It is worth noting that the type of content may vary from application to application. Nevertheless, it exhibits the content-centric behavior. In the light of the aforementioned characteristics, designing protocols for VANETs that meet QoS, and application-specific requirements, is a challenging task. In this section, we summarize the properties and applications of VANETs that may help to improve the realization and evaluation of vehicles networks.

\section{A. Properties of VANETS}

In this sub-section, we briefly discuss various distinguishing properties of VANET that differentiate it from classical MANET, as well as the challenges for the realization of vehicular networks.

1) Highly Dynamic Topology: As indicated earlier, movement in VANET is restricted by road topology; however, the individual movement topology of nodes is highly dynamic due to, at least partially, high speed and in some cases unpredictable movements [39]. As a result, the communication among nodes last for a short time, hence giving a very short time to exchange data; i.e. the connection is extremely intermittent. Indeed, this phenomenon affects the oppositeside traffic and urban scenario where multiple roads and crossroads pose extra challenges. This intermittent behavior has an adverse effect on the routing mechanisms and makes it challenging to achieve QoS guarantees for different applications.

2) Frequent Link Disruptions: As a result of high mobility, link disruption is frequent in VANET which results in unstable routing paths. Link disruptions are particularly important in case of opposite-side traffic, urban localities and nonline-of-sight (NLoS) scenarios. In the first two cases, the communication is intermittent whereas in case of NLoS, the communication is deteriorated, leading to link disruptions and network fragmentations. This problem can be mitigated by using nodes or access points along the road to improve connectivity. To date, Road Side Units (RSUs) are deployed on roadside and at the crossroads [40], [41]. However, handoff management schemes are essential to handle movement from an access point to another [42].

\footnotetext{
${ }^{1}$ Without loss of generality, we use the terms 'vehicles', 'vehicular nodes', and 'mobile nodes' interchangeably in this paper.
}

3) Mobility predictability: As compared to the parental MANET, mobility patterns in VANET are relatively predictable due to the known road topology; however, as a result of high vehicular mobility and dynamic itineraries, predicting the mobility of nodes is a challenging task. To this end, there are methods to predict mobility patterns such as toggle pattern of traffic lights, limited speed of vehicles and layout of roads' structure; however, it still needs prior knowledge of the neighbors, efficient prediction algorithms, and perfect connectivity. It is important to note that comparatively efficient prediction in VANET fosters new applications and services in VANET. It also helps location-based services to function with increased QoS [43].

4) Localized Communication: In VANET, usually vehicles are equipped with GPS and can be identified by an ID or a group ID based on their geographical location. Thus, forwarding data by targeting the individual or group of vehicles in the same geographical areas, e.g., safety applications, may help improving data delivery and reducing retransmissions. This feature of VANET also achieves other milestones such as location-based services, infotainment services, diagnostics, and so forth.

5) Propagation Models: Vehicles may move in distinct environments such as very dense traffic regime, moderate traffic regime, and sparse traffic regime. Furthermore, the scenario could be urban or highways with no obstacles at all in the surroundings. As a result of these variations, signal propagation patterns significantly vary with the passage of time making estimation of link quality and its lifetime a difficult task. This also results in poor network performance. For example, in a highway scenario, the environment is relatively simple and favors free space propagation model, while in a city, the environment is more complex and may not be free-space due to different obstacles such as vegetation, buildings, or even tall vehicles, the high density of vehicles, and their different moving direction [44], [45]. Therefore, the channel modeling in different environments is of paramount importance for efficient communication among vehicles.

6) Time Constraints: VANET applications exhibit both delay-sensitive and delay-tolerant nature. In essence, safety applications are delay-sensitive whereas infotainment applications are delay-tolerant. Since the outcome of the safety applications concern human lives, therefore, it is imperative to implement efficient, fast, responsive, trustworthy, and reliable mechanisms to enable drivers to take timely and right decision in case of designated incidents. Furthermore, in case of safety applications, reliability, and data quality are essential and critical. For example in case of an accident on a highway, the delivery of warning messages to all vehicles coming towards the site of the accident must be delivered in advance to avoid further hazards. At the same time, data quality makes sure that the messages received by the neighbors are legitimate, such messages will have dire consequences, otherwise.

7) Energy and Storage Capabilities: In contrast to MANETs and wireless sensor networks, nodes in VANETs not only have a continuous power supply but also have much better storage capabilities. In other words, recent high-end cars are equipped with sufficient computation, communication, and 
TABLE III

VANETS APPLICATIONS EXAMPLE

\begin{tabular}{|l|l|c|c|c|}
\hline Applications Type & Services Type & Local Interest & Space Validity & Time Validity \\
\hline \multirow{3}{*}{ Safety Applications } & Work Zone Warning & All vehicles & $1 \mathrm{~km}$ & Until finishing work \\
\cline { 2 - 5 } & Car accident warning & All vehicles & $500 \mathrm{~m}$ & $30 \mathrm{Sec}$ \\
\cline { 2 - 5 } & Dangerous road warning & All vehicles & $500 \mathrm{~m}$ & $10 \mathrm{Sec}$ \\
\hline \multirow{2}{*}{ Traffic Applications } & Road congestion & All vehicles & $5 \mathrm{~km}$ & $30 \mathrm{~min}$ \\
\cline { 2 - 5 } & Traffic map & Subscriber & $10 \mathrm{~km}$ & $30 \mathrm{~min}$ \\
\hline \multirow{2}{*}{ Comfort Applications } & Multimedia file sharing & Subscriber & $1 \mathrm{~km}$ & $15 \mathrm{~min}$ \\
\cline { 2 - 5 } & Commercial advertisement & Subscriber & $1-5 \mathrm{~km}$ & $1-5$ Days \\
\hline
\end{tabular}

storage resources to realize plethora of vehicular networks applications. In traditional MANET and sensor networks, the limitations stem from the resource constraints on hardware, whereas VANET does not have such limitations. As a result, network designers have the leverage to propose algorithms that can use these resources to their advantage in order to enhance network performance.

\section{B. Challenges in VANETs}

Despite the considerable amount of research results, VANET could not make it to the deployment stage due to several challenges; for instance, cost, lack of infrastructure, lack of coordination in industry, security, privacy, and consumer satisfaction, to name a few. Furthermore, these challenges also make the evaluation and implementation of network protocols more difficult and complex. The efficiency of data delivery, fast content dissemination and retrieval, and perfect connectivity in a highly dynamic mobile environment with intermittent wireless links are some of the root causes for main challenges in VANET. On the other hand, VANET uses the host-centric networking paradigm that enables end-to-end connectivity, and allows data transfer among communicating entities using IP addresses. This communication model is inherently not suitable for data-/content-oriented network where the goal of communication is data sharing and consuming regardless of who requests and who serves the request. VANET perfectly fits the criteria for content-centric networking, where the vehicles follow content-oriented paradigm by targeting the content regardless of the vehicle/node's address.

Additionally, security and privacy are other major issues that need to be assessed and resolved in the design of protocols for VANETs [46]-[48]. The security attacks in VANET can have dire consequences because it directly involves human lives, and therefore we cannot afford these attacks in VANETs [49]. Indeed, threats such as fake messages may not only compromise drivers' private information but will also cause vandalism and waste network resources by affecting communication and hence-forth leading to accidents. To mitigate such attacks, designers must incorporate trust management, resiliency, and real-time message and node authentication mechanisms [50][52].

\section{Applications of VANETs}

From a bird's view, VANET applications can be classified into three main categories: 1) safety applications, 2) traffic information applications, and 3) comfort applications. We discuss in detail, these applications and also illustrate them along with their characteristics in Table III.

- Safety Applications: This category of applications is the pinnacle of VANET, because it concerns the lives of the drivers and the passengers. The safety of both passengers and drivers are the primary concern, and therefore these applications have stringent requirements for delay, quality of service, and security. These applications include, but not limited to, cooperative collision avoidance, lane changing, information (speed limit, work zone and closed lane), and emergency warnings (accident or road condition warnings), and so forth, the information of which must be received by the drivers on time to avoid any unwanted situations that would jeopardize the lives of the occupants [53]-[55]. Such applications mostly use broadcast and flooding-based communication mechanism to disseminate messages. Hence, different communication technologies such as $\mathrm{WiFi}, 3 / 4 \mathrm{G}$, and visible light communication (VLC) are used to complement each other and to make sure that the safety-related messages have been disseminated [56]-[58].

- Traffic Information Applications: These applications are used to provide the drivers with updated traffic information, which not only enhances the view for the driver but also helps the driver avoiding traffic congestion and thereby saving both fuel and economy. The communication paradigm for such applications is unicast and has less stringent requirements than the safety-related applications. It is worth mentioning that traffic view is of two types, short-range local traffic view which can be realized through direct communication with the neighbors and long-range extended traffic view that is realized via multi-hop communications [59]-[61].

- Comfort Applications: This category aims to improve the passenger comfort and traffic efficiency. This category is also referred to as infotainment (information and entertainment). The applications in this category include exchange of weather information, gas stations location, commercial advertisements with incentives, and interactive communications such as Internet-on-the-move, movie-on-demand, and so on. These applications usually use unicast routing for communication. 


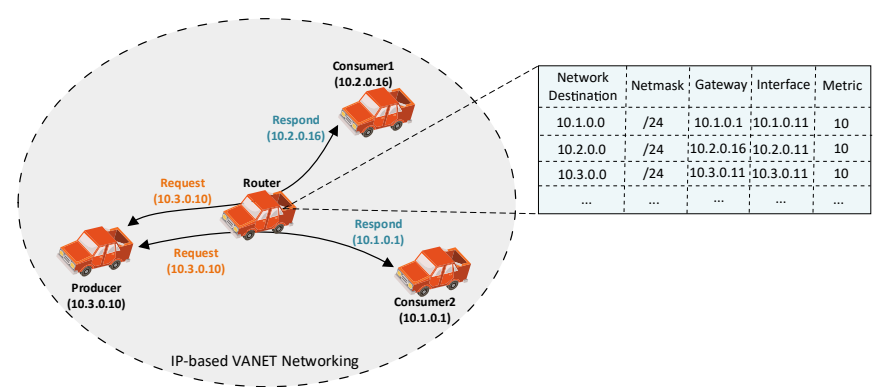

(a) IP-based VANET Networking

Fig. 2. VANET Architectures

\section{An Overview of NAMEd Data Networking}

Owning to the growing interest from academia in ICN, various architectures of ICN have been proposed in the literature [62]. Name Data Networking [63] is the most active and well-managed implementation of ICN. This section provides an overview about NDN system architecture, data structure, working principle, and the forwarding plane.

NDN architecture uses human-readable hierarchically structured names that identify any particular data to discover content, and deliver it back to the requesting consumers [64]. To this end, two types of packets are used for communication in NDN: a) interest packets that are triggered by a consumer as a request when some data is needed, and b) data packets that carry the requested data as a response to a received interest. In NDN, data packets are not sent without any previous request; such packets are considered as unsolicited and intermediate nodes drop them immediately without forwarding. However, it is worth noting that the current implementation of NDN does not allow unsolicited packets, but in future, this condition may be relaxed as VANET also includes passive push-based messages. The intermediate nodes in NDN act as relays and they are responsible to forward interest packets upstream towards content providers, deliver the content back to its consumers, and cache the content in their buffers (content store - CS) to serve future requests.

\section{A. System Architecture of NDN}

The main feature of the NDN is to avoid the hassle of addressing (IP in case of current Internet architecture). Content name is embedded in both interest and data packets. Interests are forwarded hop-by-hop using only content name until they reach the content provider or replica-node. Usually, data packet is generated and carries the same served interest name, and is delivered to the requester using the reverse path of interest packets. NDN is a receiver-driven architecture where each node maintains three main data structures:

1) Content Store $(C S)$ : The main feature of using the content name instead of host addresses is to decouple the content from its original location. Also, by applying intrinsic security mechanism, all security related measures are applied to the content itself rather than the communication channel. Hence, in-network caching can be used in NDN at intermediate nodes level. Nodes cache the content with the aim to serve future interest requests instead of forwarding the requests to

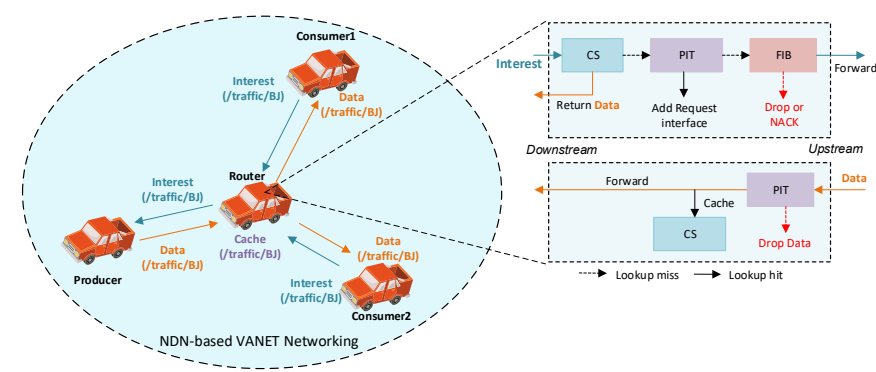

(b) NDN-based VANET Networking

the original content provider(s). This can increase the sharing probability, save more bandwidth, and reduce content retrieval time [65]. However, it is worth mentioning that efficient and reliable content replacement policy is essential for harnessing the full potential of CS in NDN [66]-[68].

2) Pending Interest Table (PIT): Every node maintains PIT in which it stores incoming interest packets until the request for that interest is satisfied by forwarding the requested content towards the consumer node. Every PIT entry contains details of the incoming interest packets i.e. requested content name, the list of interfaces at which the said interest requests have been received and the timeout interval to delete this particular entry. Nodes use PIT entries to deliver the content back to the consumers (and hence-forth called receiver-driven mechanism) without using their IP address, and enables native multicast support.

3) Forwarding Information Base (FIB): Every node in NDN also maintains FIB in which it stores information of next-hop relay nodes for reachable destination name prefixes. A node uses longest name prefix match to select suitable nexthop node to reach a destination content. FIB is used by nodes to forward interests upstream towards the content provider. The routing protocol is responsible for populating the FIB.

\section{B. Packet Forwarding Phase}

Based on the type of packet being forwarded, this phase can be further classified into: 1) interest packet forwarding, and 2) content delivery phase. Figure 2 illustrates the packet forwarding process in both IP- and NDN-based network. A term lookup hit refers to a situation when the lookup operation finds a match, and in the case of otherwise, it is called a lookup miss. The details of these phases are as follows:

1) Interest Packet Forwarding: In NDN, a consumer sends out an interest packet asking for a specific content identified by a unique name. When an intermediate node receives such interest packet, there are three possibilities. Firstly, the receiving node first checks if the requested content already exists in its local CS using the content name. If the content is in CS, the intermediate node sends back a copy of the data through the incoming interface, and the interest packet is discarded. Otherwise, secondly, the node performs an exact PIT name lookup match. If a matching entry exists, the incoming interface of this interest is appended to the already existing PIT entry. It is used for aggregating multiple interest 
requests for the same content, as the same request of other users has already been forwarded by this node and it has not satisfied the request yet. Also, this node will eventually discard the interest packet after its aggregation. When this intermediate node receives the requested data, it sends a copy to all those nodes whose interfaces are appended in the PIT entry. Thirdly, in case the intermediate node does not find any matching entry in its PIT, it will perform a lookup in FIB to find a suitable next-hop for forwarding this interest packet. If a match is found, this node will forward the interest packet to the selected node and add/create a new PIT entry with the interest name and its associated interface.

2) Content Delivery Phase: In the content delivery phase, when a node receives a data packet, it checks if the entry for the name in the data packet exists in PIT to verify whether this node had already forwarded interest packet for the content in question. If a match is not found in PIT, the node considers the data packet as unsolicited and discards it. On the other hand, if it finds a matching entry in PIT, it multicasts the data packet to all incoming interfaces listed in its PIT. Then, it discards the PIT entry as the request has already been satisfied. Furthermore, based on the caching algorithm, it also decides whether to cache a copy of the content or not.

\section{REALIZATION OF VANET THROUGH NDN}

In the recent past, vehicular networks have attracted much attention and efforts from industry as well as academia, resulting in a nice suite of protocols and standards and its commercialization is envisioned to hit the roads in near future [69]. Owing to the fact that most of the communications in VANET exhibit content-centric approach [70], efforts have been made to check the feasibility of using ICN (and favorably its particular implementation, NDN) as underlying communication architecture for VANET [71]-[73]. Consequently, various research solutions have been proposed by introducing enhancements to core NDN and by devising new design choices and guidelines to adapt NDN for VANET [74]. Different VANET communication scenarios can be modeled through NDN as a communication architecture using Interest-Data exchange model. In such an architecture, vehicles can act as consumer, provider, and/or data forwarder. At the same time, RSUs and core network elements can serve as intermediate forwarders with the caching capabilities.

In IP-based networks, different vehicles may request the same map to download or video to watch [75]. Hence, all requests must be satisfied by the original producer which is identified by its IP address. In such scenario, similar requests (with different source IP addresses) will flow from the vehicles towards the backhaul network causing congestion in the communication as well as decreasing the quality of service and network performance. The high mobility of vehicles makes the communication more challenging and may adversely affect the scalability. However, in NDN-based network, the communication is done using content-name and in-network caching feature. Decoupling the content from its location will drastically improve the content retrieval. The requested content may be satisfied from any near vehicle or RSU regardless of producer availability. Moreover, the clean architecture design of NDN handles the mobility in a simple way.

In this section, we discuss the motivation behind bringing NDN into VANETs as well as the rationale that makes NDNbased communication a suitable choice in the context of vehicular networks. Here, we focus on the pillar NDN components (i.e. content naming, forwarding, caching, security, and mobility). A detailed survey of these components and other aspects will be provided in the next sections.

\section{A. Content Naming}

Due to its simple and clean design, NDN is scalable and thus it can support huge volume of content exchange among vehicular nodes. The content naming plays an essential role in NDN-driven VANET, where naming scheme provides simple mechanism (clearly better than the current IP addressing) and can be efficiently customized to provide a name-based wide addressing range to each and every content and vehicle in VANET. Compared to IP addresses that identify and define the host location, names in NDN are specified by the application layer (from developer's perspective) and may contain different semantic information. Furthermore, it will also enable fast and efficient content routing in VANET. Different information, properties, and infotainment-related services for VANETs such as safety messages, traffic information, geographical area or spatio-temporal data can be easily described, retrieved, and stored by using human-readable NDN names.

\section{B. Content Routing and Forwarding}

NDN has a simple content discovery and data delivery mechanism as compared to current IP-based networks. As content in NDN is decoupled from its source and original location, network layer can cache and serve future requests without the need to communicate with the original producer. In other words, there is no need for a session-based communication and it does not require a continuous/in-time availability. NDN uses name-based routing where requests are forwarded using only content name in a hop-by-hop fashion to discover the content along the way (either in a cache on the path or original producer if content is not available in cache), and deliver the data back to the requester. Using cache leads to a time decoupling where content producer is not required to be always connected. Additionally, NDN supports different physical interfaces (such as WiFi, cellular, shortrange, and other interfaces) simultaneously especially when some physical interface stops working which helps in loadbalancing [76]. Furthermore, it improves the content retrieval from multiple consumers by aggregating the same interests in the PIT, which keeps track for later data delivery, and natively supports Multicast data delivery.

\section{In-network Caching}

In comparison with Internet of Things (IoT) devices, vehicular nodes in VANETs are equipped with more storage, communications, and processing resources. These capabilities make the caching process easier and more useful in VANET 
without any device constraints. On the other hand, as the content in NDN is decoupled from its original location, applying in-network caching may improve content retrieval and enhance its availability by retrieving it from the most convenient cache store, typically from the one which is nearest to the consumer node. Similarly, the use of a convenient cache replacement policy may help to keep only the popular and most used content cached [77]. As a result, interest requests might get satisfied by the CS of the intermediate nodes. Thus, the node that received the request may not forward the request to other nodes if it already has the content in CS (provided that the copy of the content is legitimate and fresh) which also reduces data latency and thus-forth network cost.

\section{Security and Mobility Support}

In session-based communication, the security refers to the communication security or security of the channel. Hosts may negotiate the use of different protocols (both cryptographic and non-cryptographic) and hashing algorithms to secure the communication. However, in NDN, security mechanisms are applied to the content itself rather than the communication channels. Therefore, the content delivery and caching may not need communication security. Moreover, due to the fact that nodes do not exchange information based on IP addresses that keep changing during mobility and joining new networks due to changing network dynamics, NDN may facilitate content access during vehicles' mobility. In case of mobile node, the node can simply re-issue unsatisfied requests. Furthermore, benefiting from the ubiquitous in-network caching, the network itself has the ability to reply to vehicles' requests regardless of the connection to the original content provider.

\section{E. Summary and Insights}

In this section, we discussed the mapping of NDN into VANET. In the light of the previous discussion, NDN is a promising communication paradigm to be used with VANET instead of the currently used host-centric model, especially for infotainment applications. In following sections, we categorize, present, and discuss the current research yielded in the field of NDN-based VANET. We cover frameworks architectures, NDN naming, routing, data discovery and forwarding, in-network caching, mobility support, and security.

\section{NDN-BASED VANET: ARCHITECTURES}

In this section, we provide a comprehensive review, and analyze of the currently available architectures for NDN-driven VANET and their key functionalities. Table IV summarizes the reviewed solutions.

\section{A. Existing NDN-based VANET Architectures}

Owing to the benefits of the NDN over traditional networking paradigm for content-based networking, efforts have been made to design new architecture(s) for realizing VANET through NDN and harness the features of ICN for VANET applications. Figure 3 illustrates a generic NDN architecture for VANET applications including components, layers, and

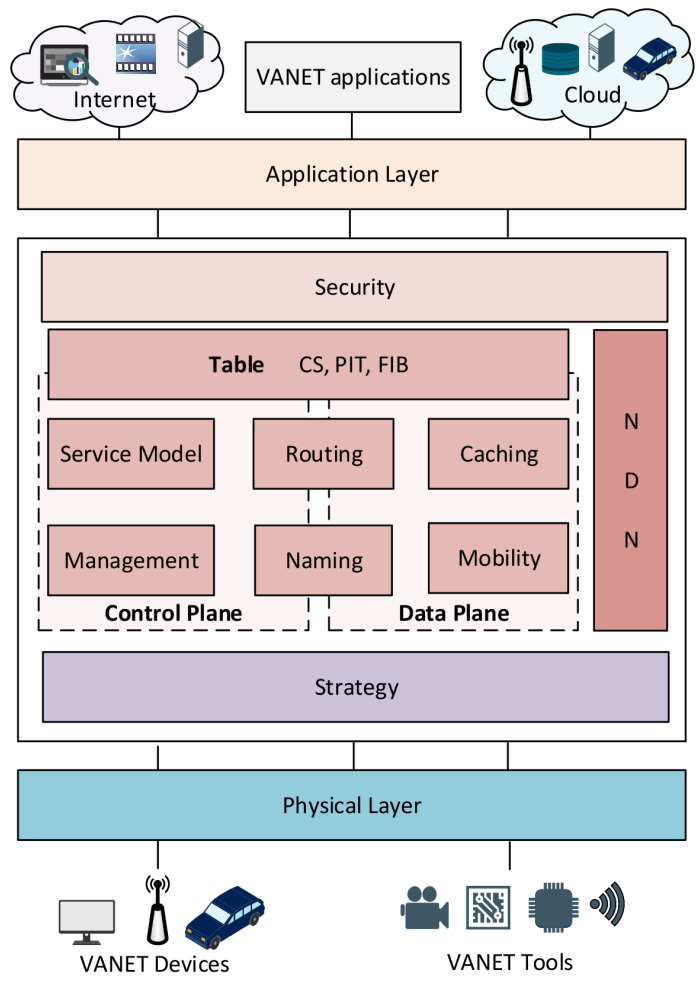

Fig. 3. A Reference NDN Architecture in VANET

functionality. The architecture consists of different layers such as physical, strategy, security, and application layers. The NDN Strategy layer aims to provide different strategies in which incorporate with the core NDN aspects (naming, caching, and routing) to provide name-based data delivery as well as handing content/device mobility (data plane). Similarly, the NDN layer may ensure both push- and pull-based communication (service model) using the Interest-Data exchange mechanism, as well as support the network management by using only content name instead of host address (control plane). During different phases and services, three data structures are involved: CS, PIT, and FIB. Whereas the security layer provides content-based security and trust model. For instance, Vehicular Name Data Networking (V-NDN) [78] architecture aims to apply NDN in VANETs by using different communication techniques such as DSRC, WiFi, and $3 \mathrm{G}$ along with different types of vehicular communications (V2X). VNDN takes benefits of NDN naming and decouples it from the communication using specific interfaces, where cars are able to use any available interface to retrieve content from any node (using in-network caching). V-NDN performs well by reducing the retransmission of interest packets and by guarantying content retrieval from the first demand via the ubiquitous in-network caching. Moreover, the authors in [78] also presented a prototype implementation of V-NDN with real experimentations. However, the proposed architecture lacks in selecting the best node for multi-home communication where well-designed forwarding strategy is needed in such context. Also, the use of geo-location data naming might not help other type of applications such as fetching certain 
TABLE IV

SUMMARY OF NDN-VANET LITERATURE

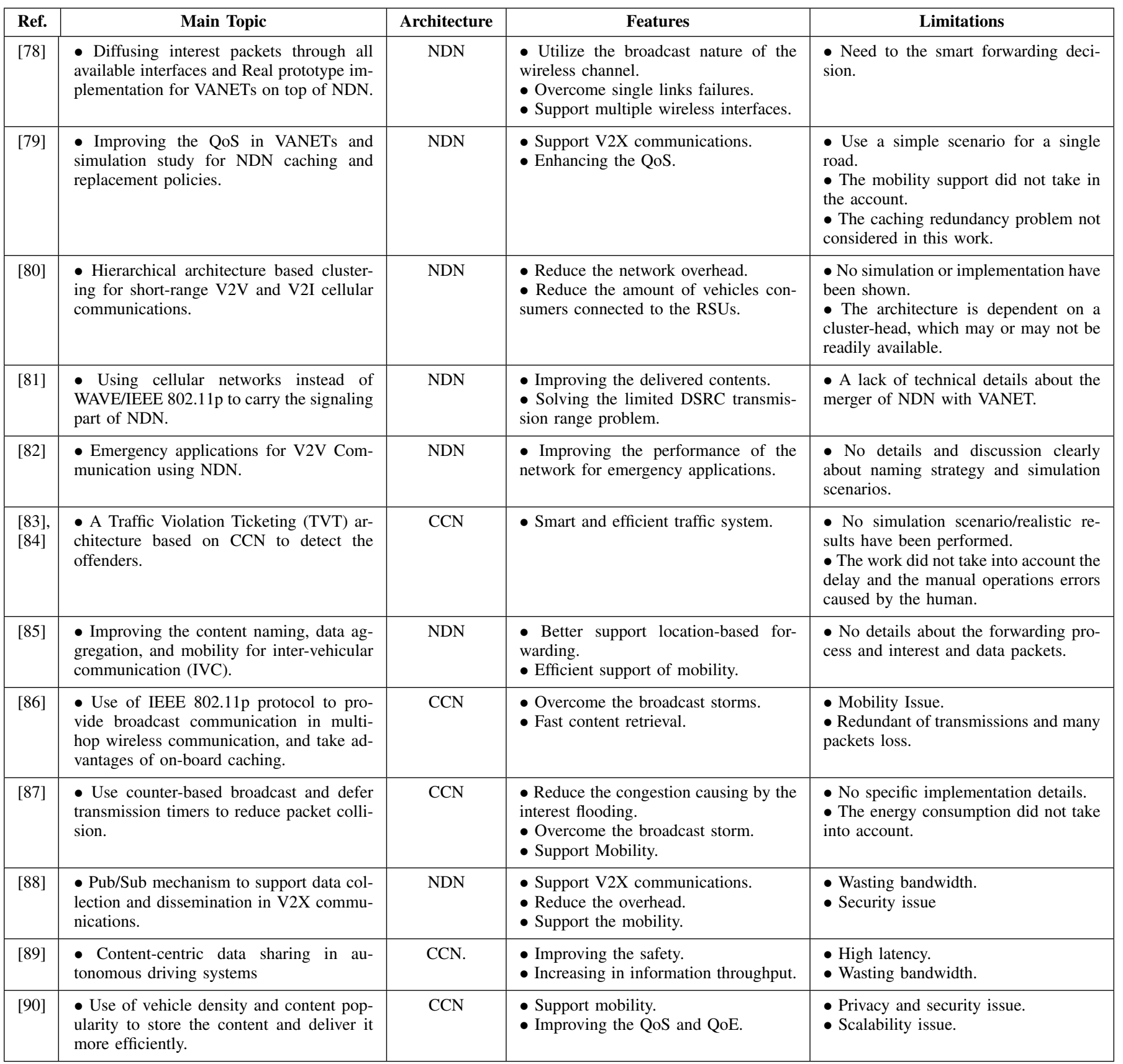

information like news (the geo-location has no significance in this type of applications). Finally, more security considerations are required in their design. In another work, Chen et al. [79] proposed Vehicular Named Data Network architecture that addresses the QoS improvement for different vehicle communication (V2I, V2R, and V2V). The authors focus only on the content popularity-based caching scheme by analyzing the performance of the cache decision and replacement policy for NDN.

A hierarchical architecture is proposed in [80] to distribute the content in VANETs by using clustering on top of NDN architecture. This design exploits both short-range V2V and
V2I cellular communications. The use of clustering may limit the number of broadcast messages consequently decreasing the number of retransmissions, number of consumers, and respectively allowing rapid content retrieval in the network, by targeting only vehicles that are in the cluster. However, the dynamic mobility of vehicles may affect the overall performance. Another similar work in [81] addresses the use of cellular networks instead of WAVE/IEEE 802.11p to carry the signaling part of NDN. This works address the limitations of DSRC transmission range.

Ho et al. [82] proposed emergency application for $\mathrm{V} 2 \mathrm{~V}$ communication using NDN. This architecture uses different 
naming schemes based on different types of emergency applications and location information that may allow the vehicles to quickly determine whether these messages fit for their interest or not. The authors manipulated the NDN data structures, i.e. PIT, FIB, and CS to support the architecture's communication principles. However, these changes in NDN design are not desirable as they violate the NDN native forwarding process and would thus-forth need modification in NDN engine for each vehicle. Similarly, Ahmed et al. [83] proposed traffic violation ticketing architecture that applies the basics of VCCN and allows traffic officials to detect the offenders and issue them relevant tickets without any human interference. However, this architecture is yet to be implemented. In [84], the authors extended their previous work [83] and proposed SmartCop, a smart traffic violations ticketing system for VNDN, by providing future research directions for implementing and improving the SmartCop system in real testbed environments.

Yan et al. [85] extended the basic NDN model for vehicular information networks to support location-based forwarding for both pull and push-based communications. They also focused on interest segregation to adapt NDN with large-scale but small-sized content retrieval, data aggregation, and distributed mobility management based on content name and car moving information. However, for this model to work with commercial VANET, a prototype implementation is essential to have more insights to the technical concerns such as forwarding strategies of both interest and data packets.

CRoWN [86], a Content centRic netWorking in vehicular ad hoc Networks, is a CCN-based VANET architecture. CRoWN uses content-centric communications on top of IEEE 802.11p physical and Media Access Control layers to provide broadcast communication on wireless medium, multi-hop V2V communications, and on-board caching. Similarly, Amadeo et al. [87] proposed a similar architecture namely ContentCentric Vehicular Network based on their previous work. This framework uses a simple counter-based broadcast approach coupled with defer transmission timers in order to reduce packet collision.

Drira et al. [88] extended NDN with a publish-subscribe capability to support data collection and dissemination in V2X communications. The enhanced NDN reduces the number of packets exchanged in the network for subscriber vehicles by allowing the subscribers to receive many data packets for one subscription without sending the interest packet in continuous manner, which is achieved by using persistent interest that kept active in PIT entry for long time. In addition to interest and data packets, the authors also proposed a new packet header with different message structures and procedures to support un/subscription and publishing operations. The new introduced fields include time-stamp, validity time, and node type. Although this architecture is a good integration of pub/sub in NDN-VANET; nevertheless, the underlying changes in native NDN design and packet types are not yet tested for suitability in large-scale deployment. Furthermore, Kumar et al. [89] proposed a system for autonomous driving that uses contentcentric patterns in order to allow cars to obtain sensory information, which is collected by other vehicles in a similar manner to how obtaining information from their local sensors.
This approach also uses the multi-resolution system to access road regions. However, the latency overhead incurred by the proposed scheme is a major drawback. Additionally, it is difficult to extend the system with other V2V communications and support other applications due to its increased latency, extreme time sensitivity, and required latency to discover the data routing path. To partially counter this issue, $\mathrm{Su}$ et al. [90] proposed a framework based on content-centric vehicular network in order to deliver content more efficiency. Contents are stored in CS based on two factors: 1) vehicular density and 2) content popularity. Moreover, pending interests are refreshed based on transmission ratio and network topology. Authors also determined the location of a content-centric data/content unit based on the forwarding information in the FIB. However, this work did not discuss the underlying security, privacy and mobility issues, which are essential in future VANET communications.

Despite the fact that the aforementioned architectures envision the integration of NDN and VANET, a more generalized unified architecture is required that merges native NDN communication with VANET communication in a holistic way, which encompasses all aspects of VANET applications. It is important to emphasize here that VANET supports different applications with varying communication, storage, computation, security, and privacy requirements. Furthermore, these applications have different design requirements and context. Moreover, the merger and NDN-based solution for VANET should introduce minimum enhancements to both technologies to utilize their full potential.

1) Performance Evaluation Overview of NDN-based VANETS: In addition to the architectural design of NDNbased VANET, the performance evaluation of applications realized through these architectures is of paramount importance and helps determining the feasibility of these architectures. To date, various efforts have been made to evaluate the performance of NDN-VANETs [91]-[95] through different performance metrics as discussed below.

Amadeo et al. [91] analyzed the transport-level issues in CCN architecture on top of the IEEE 802.11p access technology. Then, they proposed a content segmentation scheme coupled with an interest retransmission policy to ensure reliability and improvement in content delivery. The round trip time estimation is used in this architecture as a reference for retransmission, and is carried out according to exponential weighted moving average. On the other hand, Kuai et al. [92] examined the broadcast performance of V-NDN by using the ORBIT testbed ${ }^{2}$. In this work, a real V-NDN testbed is used and altered for the experiments needs. This study shows that in the high-density network, performance of the V-NDN degrades when the loss ratio increases. This phenomenon is the expected behavior of the network, but more investigation is needed to adapt NDN-based VANET to address this issue. Yu et al. [95] studied the benefits of applying ICN in VANET through caching and data selection policies. Their results show that the pervasive caching is the most important benefit that

${ }^{2}$ ORBIT testbed. Available: www.orbit-lab.org 
directly affects the data retrieving from the nearest cache store instead of data producer.

One of the best and affordable ways to assess the performance of NDN-based VANET is to conduct simulations. In other words, a simulation-based study on the applicability of NDN in vehicular environment shows the effectiveness of NDN in VANETs in multi-hop communication to exchange messages for a variety of VANET related services [93]. To this end, Bouk et al. [94] examined the distinctive aspects of NDN over VANETs in terms of interest forwarding, interest drop, interest satisfaction, difference between interest and data propagation path, number of PIT entries, and data propagation delay. The authors showed that by utilizing multi-path propagation, there is unpredictable interest satisfaction delay as well as propagation of unnecessary redundant copies of interest/data packets. Therefore, pending interest information with varying duration requires an efficient interest/data forwarding strategy. Furthermore, it is important to highlight that NDN does not maintain a separate transport layer as IP based networks do. We can notice from the above performance evaluation studies that none of them target NDN transport issues. Most of the transport layer features such as reliability and retransmission are embedded in NDN forwarding plane. Thus, we believe that more in-depth investigative research is required to address these issues in forwarding schemes according to the requirements of various VANET communication models.

\section{B. Summary and Insights}

In this section, we surveyed the existing efforts on the integration of NDN and VANET. We particularly covered the proposed architectures and performance-related studies. We notice that the majority of the proposed solutions target specific aspects of VANET such as V2V, V2I, or specific applications such as traffic violation ticketing, etc. NDNbased VANET efforts separately focus on issues like naming, forwarding, and publish-subscribe without considering the required aspects together as part of a single architecture. To this end, a holistic architecture that covers all VANET scenarios through NDN, is essential. Furthermore, we also note that there is a lack of work in the real implementation of NDN-based VANET architecture. More in-depth investigation is required from the academia and industry to address these issues.

\section{NDN-VANET NAMING SCHEMES}

Naming is the pinnacle of NDN that differentiates it from traditional networks. NDN names should be globally unique, secure, location-independent, and human-readable [106], [107]. Designing a naming scheme must satisfy all these aforementioned requirements. Current NDN naming schemes can be classified into the following four categories: hierarchical, flat, attribute-value based, and hybrid names. We further discuss these categories below.

Hierarchical names are similar to Uniform Resource Locator (URL) like structure. While flat names are obtained by applying hash algorithms to the whole or part of the content. Attribute-value name has a collection of attributes, whereby each attribute consists of a name, type, and a set of possible values all together representing a single content. Finally, hybrid names may merge two or more types.

In the following, we discuss the existing naming solutions in the context of VANET. To the best of our knowledge, and due to the limitations of flat and attribute-values naming schemes, existing NDN-VANET naming efforts focus primarily on hierarchical and hybrid names. Table V summaries the existing naming schemes.

\section{A. Hierarchical Naming}

Hierarchical names support aggregation [108], [109] where names are aggregated based on the longest prefix match. Hence, such schemes may reduce the size of the forwarding table, provide fast name lookup, and support a scalable network. However, they lack in the persistence support; a name structure modification would be required in case any change occurs in the content hierarchy, especially for changes in topological names by the content owner or provider.

Wang et al. [96] proposed a data naming structure for $\mathrm{V} 2 \mathrm{~V}$ traffic information dissemination. Names are represented in the following format: /traffic/geo location/timestamp/datatype/nonce. In this format, the traffic component contains the application ID, geo-location uses the format of road ID/direction/section number, timestamp represents a time period, data-type component indicates the meaning of the data itself, and nonce is used to avoid redundancy of packets. The use of data-type enables data to be exchanged in more efficient manner in a highly dynamic environment with intermittent connectivity among vehicles.

Pesavento et al. [97] designed a hierarchical naming scheme for vehicular networks that embeds geographic areas information by using encoding algorithm, and converts the coordinates of location $(x, y)$ to become a separate name component using an agreed-upon pairing function $\left(/ \mathrm{ndn} / \mathrm{bit} /\right.$ parking $\left./ \ldots / \mathrm{c}_{\mathrm{n}}\right)$. The objectives of this scheme are to improve the in-network caching, increase the cache hit ratio, and liberate the interest and data packets forwarding without involving another specific application in the process. Similarly, a hierarchical naming scheme for the vehicular information network is proposed in [85]. This scheme supports both pull-based and pushbased communication. The name structure has mainly three fields: the first field includes destination location $(D-L)$ and the requested data types, the second field contains the source location $(S-L)$ and the data provided by this consumer, and the last field is the next location along the direction from $S$ $L$ to $D$ - $L$. Furthermore, this naming scheme also addresses the challenges of interest segregation/data aggregation and mobility management in vehicular networks.

Drira et al. [98] designed new query names patterns for collecting data in V2X communication. The naming scheme contains three components which are prefix/query, key, and value. The key component represents the type of data source node. The value is composed of three sub-components, conditions that is used to select the nodes that represent a logical expression such as speed, lane, position, etc. The second sub-component is selected key that is embedded in 
TABLE V

SUMMARY OF EXISTING NDN-VANET NAMING SCHEMES

\begin{tabular}{|c|c|c|c|c|c|}
\hline Ref. & Name Format & Comparison & Parameter Evaluation & Simulation Tools & Year \\
\hline \multicolumn{6}{|c|}{ Hierarchical Naming Schemes } \\
\hline [96] & /traffic/geolocation/timestamp/datatype/nonce & No comparison & - Not mentioned & No Simulations & 2012 \\
\hline [97] & /ndn/ucla/parking/::: /cn & No comparison & $\begin{array}{l}\text { - No. of satisfied inter- } \\
\text { ests } \\
\text { - CDF for interest satis- } \\
\text { faction time }\end{array}$ & NS3, ndnSIM & 2013 \\
\hline$[85]$ & $\begin{array}{l}/ \mathrm{D}-\mathrm{L}(1): \ldots: \mathrm{D}-\mathrm{L}(\mathrm{n}): \text { end/type }: \ldots: \text { type }: \text { end } / \\
\mathrm{S}-\mathrm{L} / \text { type\&data }: \ldots: \text { type\&data }: \text { end } / \text { next }: \ldots: \text { next }: \\
\text { end/ }\end{array}$ & No comparison & $\begin{array}{l}\text { - Aggregation time } \\
\text { - Number of locations }\end{array}$ & Matlab & 2014 \\
\hline [98] & $\begin{array}{l}\text { query/Node_Selection/Conditions/Selected_Key/ } \\
\text { Time_Condition }\end{array}$ & No comparison & $\begin{array}{l}\text { - Throughput } \\
\text { - Latency } \\
\text { - Number of messages }\end{array}$ & $\begin{array}{l}\text { NS3, ndnSIM, } \\
\text { SUMO }\end{array}$ & 2014 \\
\hline [99] & /Category/Service_name/Additional_info/ & $\mathrm{CCN}$ & $\begin{array}{l}\text { - The response time } \\
\text { - The completion rate }\end{array}$ & $\begin{array}{l}\text { Qualnet with } \\
\text { VanetMobiSim }\end{array}$ & 2015 \\
\hline [100] & $\begin{array}{l}\text { /application/geo_reference/temporal_field/ } \\
\text { nonce/ }\end{array}$ & $\begin{array}{l}\text { - First contact } \\
\text { - P-Random } \\
\text { rate }\end{array}$ & $\begin{array}{l}\text { - Average Number of } \\
\text { Hops } \\
\text { - Average satisfied delay } \\
\text { of interest } \\
\text { - Data delivery delay }\end{array}$ & ONE & 2016 \\
\hline [101] & $\begin{array}{l}\text { /application_prefix/datatype/data_location/ } \\
\text { name_marker/vehicle_name/timestamp }\end{array}$ & No comparison & $\begin{array}{l}\text { - Consumer delay: } \\
\text { - Data Fetching Delay } \\
\text { - Certificate Fetching De- } \\
\text { lay } \\
\text { - Data Validation Delay } \\
\text { - Producer delay: } \\
\text { - Data Signing Delay } \\
\text { - Certificate Sending De- } \\
\text { lay }\end{array}$ & $\begin{array}{l}\text { Implementation } \\
\text { using } \\
\text { Raspberry } \\
\text { Pi }\end{array}$ & 2017 \\
\hline [102] & $\begin{array}{l}\text { Top - Level_Category/Class_Category/Application } \\
\text { /Primary_Identifier/Contextual_Identifier/ } \\
\text { Additional_Tags/ }\end{array}$ & pNDN & $\begin{array}{l}\text { - Delivery Rate } \\
\text { - Latency } \\
\text { - Total transmitted data } \\
\text { packets } \\
\text { - Effects of Beacon fre- } \\
\text { quency }\end{array}$ & $\begin{array}{l}\text { OMNeT++, } \\
\text { SUMO }\end{array}$ & 2017 \\
\hline \multicolumn{6}{|c|}{ Hybrid Naming Schemes } \\
\hline [103] & $\begin{array}{l}\text { hmn ://traffic/highway/safty/video } \\
: \text { 629d5f3f1f0a112f } \\
: / \text { Time/Birate/Public/Emergency }\end{array}$ & No Comparison & $\begin{array}{l}\text { - Start-up delay } \\
\text { - Playback freezing }\end{array}$ & NS3, ndnSIM & 2014 \\
\hline [104] & vhn ://us/nv/lv/smith/NVD0001/text/.../uSwjkHZ & No Comparison & Not mentioned & No Simulations & 2014 \\
\hline [105] & 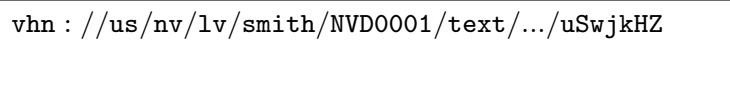 & $\begin{array}{l}\text { - Simple Trie } \\
\text { - NLAPB }\end{array}$ & $\begin{array}{l}\text { - Prefix add time } \\
\text { - Prefix delete time } \\
\text { - Memory consumption }\end{array}$ & $\mathrm{C}++$ & 2015 \\
\hline
\end{tabular}

the data packet. The last sub-component in value is time conditions that describe the interval and the period among observations. Another hierarchical naming scheme for VANET is proposed in [99] where the naming convention is organized as /Category/ServiceName/AdditionalInfo. Category represents the type of data based on the popularity factor and share-ability feature, and is used to decide the routing approach. ServiceName identifies a service that is provided by multiple nodes, and AdditionalInfo represents the content identifier. An example of this scheme, name for a map of Beijing, Haidian District can be defined as /type_a/map/beijing/haidian/.

Similarly, a hierarchical naming scheme has been proposed in [100], it has the following naming format: /application/geo - reference/temporal - field/nonce. The Application component indicates the type of application that is associated with the content. The geo-reference represents the ID of 'position of interest'. The temporal-field is represented as the start-time/end-time in interest packet, and indicates as the time of published content in data packet, where the start-timelend-time is the interval time that need to get the data. The nonce is used to identify different data providers. Chowdhury et al. [101] proposed a similar scheme by introducing a hierarchical names for trust model to verify whether the data is produced by autonomous vehicles or not. The naming scheme is represented as /application - prefix/datatype/data location/name - marker/vehicle - name/timestamp. The application-prefix component represents the name of application that produces the data, datatype component 
represents the kind of data that is produced, and data-location is used to specify the geo-location of the content. As the location may have multiple name components, the component name-marker is used as a tag to identify from where the vehicle name starts. The vehicle-name is represented as /manufacturer - name/vehicle - name, where former sub-component identifies the vehicle's manufacturer and the latter identifies the vehicle. Finally, the timestamp component represents time period. The difference between this work and the previous work [96] is that the current scheme contains the name/pseudo-name of vehicle that produced the data/content, in which it aims to bind the data with its origin vehicle producer, and provide authenticated data.

In [102], the authors introduce a service-based system architecture for NDN in vehicular networks. This architecture is based on using a global naming scheme. This naming scheme includes top level category that represents one of three main categories: safety services, transit information, and infotainment. Class category is used for content prioritization. Application that identifies the type of services and grouped into class categories, and finally, Content Identifiers and MetaInformation that are used to limit inconsistencies that produced from different requesting information from neighboring vehicles.

In summary, hierarchical names can be a good choice for content naming in NDN; however, these naming schemes are difficult to use as they may get lengthier and may also have variable length according to the application and semantic design. Thus, lookup efficiency may be decreased and thusforth may affect the overall performance of the network. To overcome these challenges, recent research works are focusing on hybrid naming schemes as discussed in the following subsection.

\section{B. Hybrid Naming}

In order to get the best features from two naming schemes (hierarchical, flat, or attribute-value based), hybrid naming schemes are used that inherit the advantages of both schemes. In essence, hybrid naming mechanisms have more advantages such as improved scalability, performance, and security [110].

To this end, various hybrid naming schemes have been proposed for NDN [111]-[114]; however, the current hybrid schemes lack in consideration for NDN-based VANET. Quan et al. [103] designed a hybrid naming scheme that combines hierarchical, hash, and attribute-value names for multimedia contents in VANETs to improve the Quality of Experience (QoE) of multimedia streaming. The format of naming includes three parts: Hierarchical Routable Prefix (HRP), Flat Content Identifier (FCI), and Primary Attribute Labels (PALs). The HRP represents the hierarchical multimedia naming and used for routing and name aggregation, whereas FCI is the hash of whole or part of the content, and used to accelerate the content and caching discovery. Finally, PALs are the attributes information for the content such as timeliness, caching strategy, and priority level that can be used for multiple aggregations requests. The three parts HRP, FCI, and PALs are separated by ":" while all name components with content attributes are separated by "/". However, this naming mechanism has inherent disadvantages such as variable length of the name and long prefix lookup time.

Similarly, Bouk et al. [104] proposed a hybrid naming scheme for Vehicle ICN, which uses the collective characteristics of hierarchical and hash-based naming schemes. Name is divided into three parts: the first part is named as Scheme and it represents the vehicular network or vehicular identifier. The second part is the Hierarchical part and contains information and details about the node originating and the content itself. The second part helps in name aggregation and simplifies the routing decisions. The last part is the Flat part, which is the hash of the item (owner or signature of the owner) and is presented in Base64 format. The flat part ensures contents integrity. However, the current scheme is theoretical and needs in-depth feasibility study. Further, the proposed scheme has several issues including the variable length of the name, where no constraint exists on the name length, which will likely generate a large prefix table and thereby incurring long prefix lookup time. Bouk et al. extended this work with efficient Compact Trie management scheme for VCCN in [105]. Furthermore, they analyzed the simulation results for VANETs which show that the lookup time and memory management are improved for the proposed scheme.

Through hybrid naming scheme, various advantages of individual schemes can be combined, expecting to improve content naming, lookup, and forwarding process. However, an indiscriminate combination may create more complex schemes. Hence, careful design choices are needed to provide a unified scheme for NDN-based VANET.

\section{Summary and Insights}

In this section, we investigated and analyzed NDN-based naming schemes applicable for VANET applications. From the discussion, we can see that hierarchical naming and hybrid schemes gained more attention from researchers as compared to flat and attribute-based naming schemes. However, it is worth noting that we cannot overlook the advantages of flat and attribute-value scheme. A scalable and unified naming scheme should incorporate advantages of each and every aspect, where global hybrid naming schemes are still essential for VANETs.

\section{ROUTING, DATA Discovery, ForWARding AND CONTENT DISSEMINATION IN NDN-BASED VANET}

NDN enables users to access content and services by using hierarchical unbounded names regardless of the content location. The core routing of NDN uses only names to discover and deliver the content [106]. NDN routing plane aims to find path towards availability of the requested content, whereas the forwarding plane forwards interest and data packets towards the next hop nodes. In this section, we present a detailed overview of the existing routing and data forwarding solutions proposed in the context of VANETs. Based on their working principles, we classify the existing strategies into eight categories, as shown in Figure 4, and summarize them in Table VI. 


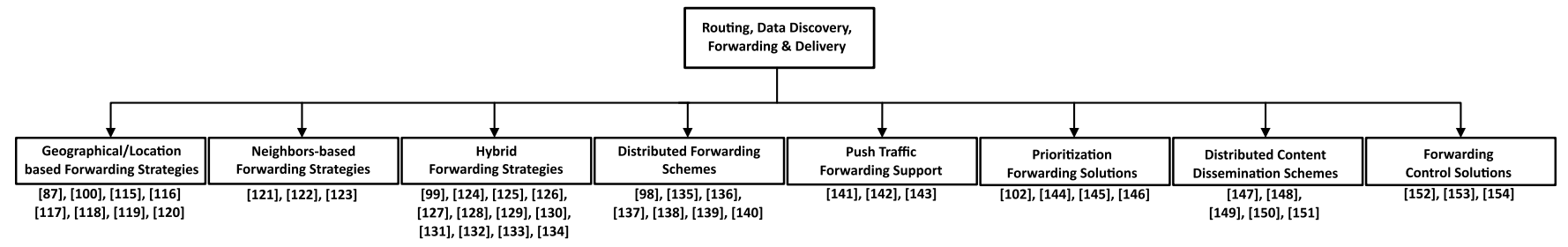

Fig. 4. Existing NDN-VANET Forwarding Schemes.

TABLE VI

SUMMARY OF EXISTING NDN-VANET FORWARDING SCHEMES

\begin{tabular}{|c|c|c|c|c|}
\hline Category & Ref. & Summary & Benefits & Limitation \\
\hline $\begin{array}{l}\text { Geographical, Lo- } \\
\text { cation based For- } \\
\text { warding }\end{array}$ & $\begin{array}{l}{[115],} \\
{[116],} \\
{[87],} \\
{[117],} \\
{[118],} \\
{[119],} \\
{[120],} \\
{[100]}\end{array}$ & $\begin{array}{l}\text { - The use of geo-location information } \\
\text { coupled with defer timer in order to } \\
\text { choose the best next relay nodes }\end{array}$ & $\begin{array}{l}\text { - Minimize the collision probabil- } \\
\text { ity } \\
\text { - Limit the interest flooding }\end{array}$ & $\begin{array}{l}\text { - Scalability issue } \\
\text { - Predicable mobility model } \\
\text { - Wasting bandwidth by beacon } \\
\text { messages }\end{array}$ \\
\hline $\begin{array}{l}\text { Neighbors-based } \\
\text { Forwarding }\end{array}$ & $\begin{array}{l}{[121],} \\
{[122]} \\
{[123]}\end{array}$ & $\begin{array}{l}\text { - Select the best relay node based } \\
\text { on node properties and data structures } \\
\text { that are stored in each vehicle and ex- } \\
\text { changed periodically by beacon mes- } \\
\text { sages }\end{array}$ & $\begin{array}{l}\text { - Select the best forwarder } \\
\text { - Mitigate the broadcast storm } \\
\text { problem }\end{array}$ & $\begin{array}{l}\text { - Large collision and congestion } \\
\text { caused by selecting a single for- } \\
\text { warder by multiple vehicles } \\
\text { - Large overhead caused by beacon } \\
\text { messages }\end{array}$ \\
\hline $\begin{array}{l}\text { Hybrid Forwarding } \\
\text { Strategies }\end{array}$ & $\begin{array}{l}{[99],} \\
{[124],} \\
{[125],} \\
{[126],} \\
{[127],} \\
{[128],} \\
{[129],} \\
{[130],} \\
{[131],} \\
{[132],} \\
{[133],} \\
{[134]}\end{array}$ & $\begin{array}{l}\text { - Hybrid forwarding schemes that used } \\
\text { different geo-location information and } \\
\text { nodes properties to select the corre- } \\
\text { sponding forwarding nodes }\end{array}$ & $\begin{array}{l}\text { - Achieve efficient and reliable } \\
\text { communication } \\
\text { - Mobility support }\end{array}$ & $\begin{array}{l}\text { - Storage overhead } \\
\text { - Bandwidth overhead by beacon } \\
\text { messages }\end{array}$ \\
\hline $\begin{array}{l}\text { Distributed } \\
\text { Forwarding } \\
\text { Schemes }\end{array}$ & $\begin{array}{l}{[98],} \\
{[135],} \\
{[136],} \\
{[137],} \\
{[138],} \\
{[139],} \\
{[140]}\end{array}$ & $\begin{array}{l}\text { - Distributed interest forwarder selec- } \\
\text { tion that chooses the best relay node } \\
\text { based on shared information such as } \\
\text { speed, direction, and position }\end{array}$ & $\begin{array}{l}\text { - Mitigate the interest broadcast } \\
\text { storm } \\
\text { - Solve the disconnected link prob- } \\
\text { lems }\end{array}$ & $\begin{array}{l}\text { - Bandwidth overhead by beacon } \\
\text { messages }\end{array}$ \\
\hline $\begin{array}{l}\text { Push Traffic For- } \\
\text { warding Support }\end{array}$ & $\begin{array}{l}{[141],} \\
{[142]} \\
{[143]}\end{array}$ & $\begin{array}{l}\text { - Push-based critical data forwarding } \\
\text { scheme for data dissemination to al- } \\
\text { low vehicles to broadcast an emergency } \\
\text { messages when necessary }\end{array}$ & $\begin{array}{l}\text { - Ability to broadcast an emer- } \\
\text { gency messages without any pre- } \\
\text { vious interest }\end{array}$ & - Predicable mobility model \\
\hline $\begin{array}{l}\text { Prioritization For- } \\
\text { warding }\end{array}$ & $\begin{array}{l}{[144]} \\
{[145]} \\
{[102]} \\
{[146]}\end{array}$ & $\begin{array}{l}\text { - Using the prioritization mechanism } \\
\text { coupled with the timer to favor the } \\
\text { transmission of certain packet types } \\
\text { over others and guarantee QoS }\end{array}$ & $\begin{array}{l}\text { - Improve the Quality of Service } \\
\text { - Manage information dissemina- } \\
\text { tion }\end{array}$ & $\begin{array}{l}\text { - Need to know the global content } \\
\text { popularity } \\
\text { - Limited efficiency when the net- } \\
\text { work load is high }\end{array}$ \\
\hline $\begin{array}{l}\text { Distributed } \\
\text { Content } \\
\text { Dissemination }\end{array}$ & $\begin{array}{l}{[147],} \\
{[148]} \\
{[149]} \\
{[150]} \\
{[151]}\end{array}$ & $\begin{array}{l}\text { - A content distribution mechanism } \\
\text { based on the benefit of multiple paths }\end{array}$ & $\begin{array}{l}\text { - Improve the reliability and robust } \\
\text { distributed dissemination mecha- } \\
\text { nism }\end{array}$ & $\begin{array}{l}\text { - Huge amount of redundant data } \\
\text { while distributing content } \\
\text { - Storage overhead }\end{array}$ \\
\hline $\begin{array}{l}\text { Forwarding } \\
\text { Control Solutions }\end{array}$ & $\begin{array}{l}{[152],} \\
{[153]} \\
{[154]}\end{array}$ & $\begin{array}{l}\text { - A controlled data packets broadcast } \\
\text { storm using the hop counter }\end{array}$ & $\begin{array}{l}\text { - Limit the additional copies of } \\
\text { data packets }\end{array}$ & - Predictable mobility model \\
\hline
\end{tabular}

\section{A. Location based Forwarding}

In geographical- or location-based forwarding schemes [155], the interest packets are guided by geo-location infor- mation and are forwarded by the sender to those intermediate nodes that are moving in the same direction. Such forwarding strategies may tackle the issue of high-speed mobility by 
limiting the number of transmitted packets.

In [115], the authors proposed a simple application for traffic information dissemination for $\mathrm{V} 2 \mathrm{~V}$ communications. The application uses geo-location information from content naming to forward interest packet and uses defer timers to control the packet redundancy. Timers are randomly selected by neighboring vehicles that received the interest packet, when they receive the same interest at the second time, they schedule the transmissions of data packets at different times. However, geo-location information needs to be included in the data packets, which means altering the standardized data structure.

Similarly, Tarroumi et al. [116] enhanced the previous architecture and proposed a new mechanism of relay node selection in order to reduce the collision and the error rate, as well as to improve the QoS in the network. The objective of this mechanism is to select the next-node hop within the middle, and at the border of transmission range instead of selecting the farthest node. A similar work based on defer timer has been proposed in [87] to reduce the probability of collision. The nodes use two defer timers for forwarding interest and data packets. Doing so also helps in reducing retransmissions as nodes overhear packets transmitted by their neighbors. If a node overhears the same packet being transmitted by other nodes, it aborts the re-broadcast of an interest packet.

To reduce network overhead, Yu et al. [117] designed Last Encounter Content Routing that uses the last encounter information discovery and opportunistic geographical routing. Each vehicle maintains Last Encounter List where information about the neighbors and the content location are updated during every encounter. When a vehicle receives an interest packet and its name gets matched with an entry in its Last Encounter List (list of content locations), it will forward this interest packet through geo-routing instead of flooding. Otherwise, it uses flooding to disseminate the interest packet.

In [118], [119], a geo-based forwarding strategy for Urban VANETs based on NDN is proposed. To direct the interest packet geographically, every node maintains a neighbor table that contains IDs of its neighbor vehicles along with their position. This table is updated periodically using beacon messages. Also, to enhance packet delivery reliability, nodes use a timer-based forwarding decision mechanism and multipath forwarding by taking caching benefits into account. Moreover, [119] addresses the caching redundancy problem by proposing heuristic strategies, which are a random selection caching heuristic strategy and density-aware strategy. The first strategy consists of caching the solicited received data with a predefined probability, while the second consists of selecting only vehicles (along with the forwarding path) that have a uniform spatial distribution. These two heuristic strategies may reduce unwanted cache copies. Similarly, another work [120] proposes a mechanism named Navigo, that combines content's name with producers' geographic area to guide interest forwarding. The goal is to reduce the flooding of interest packet. Authors defined a name-space by using the Military Grid Reference System and mapping for each geolocation. Furthermore, they introduced GeoFace field in the FIB table that binds the name prefix to the geo-location. Furthermore, Navigo uses a Link Adaptation Layer algorithm to calculate the shortest path and handle interest timers.

A geographic opportunistic forwarding strategy for V-NDN has been proposed in [100] aiming to choose better next relay nodes by using geographic location of the Position of Interest and vehicle trajectories. The authors also introduced a new forwarding strategy for interest and data packets. On the contrary, it is not possible to assess the effectiveness of the proposed protocol as authors did not compare the performance of their proposal with any native V-NDN protocol.

From the above discussion, we can conclude that geolocation based forwarding schemes may help reduce the number of retransmitted interest packets and hence avoiding interest flooding. However, most of the afore-mentioned solutions either define new data structure such as Last Encounter List, Neighbor Table, or are based on the use of beacon messages. These schemes also include content names with the location of provider which limit the use of in-network caching, and cause major changes in the FIB table structure. The change of native NDN architecture is not preferable, especially for large-scale deployment.

\section{B. Neighbors-based Forwarding Strategies}

The forwarding decision in Neighbors-based forwarding is based on the selection of an appropriate neighbor based on different metrics such as most satisfied neighbors, mobility factor (e.g., the mobility speed, the mobility prediction, etc), or caching probability.

Ahmed et al. [121] proposed a Robust Forwarder Selection mechanism that allows the consumer to select only one vehicle among the neighboring vehicles to forward the interest packet. This scheme uses two data structures: 1) Neighbors Satisfied List (NSL), and 2) Recent Satisfied List (RSL). NSL contains information about satisfied interests that a node gathers by exchanging RSL with its neighboring vehicles. This information is exchanged periodically using beacon messages to keep the NSL updated and select the potential interest forwarder. This solution is not suitable in case of single forwarder that is selected by multiple vehicles which may lead to collisions and congestion. Also, a considerable overhead is incurred by periodically exchanging beacon messages.

Kuai et al. [122] proposed a Density-Aware Delay-Tolerant interest forwarding strategy in vehicular NDN to improve packet delivery ratio. Each vehicle maintains a list of its neighbors along with their location and timestamp. These information are updated on-the-air during receiving an incoming packet. Every node discovers location information of its neighbors' vehicles by exchanging periodic beacons messages. This scheme also uses a rebroadcast deferring timer to mitigate the broadcast storm. Furthermore, Lin et al. [123] designed a Reliable Data Forwarding Scheme with the aim to provide a reliable end-to-end connection, while considering the mobility issues. The consumer vehicle in this scheme selects the neighbor vehicle with the farthest distance from it as relay. The relay vehicle then disseminates the message within its vicinity after setting a waiting timer.

In summary, Neighbors-based forwarding strategies reduce retransmissions of interest packets in the network. However, 
the majority of them require the nodes to be equipped with GPS unit or some other localization techniques. Moreover, nodes have to maintain an up-to-date neighbors' list along with their current location which will be difficult in case of unstable links due to high mobility and intermittent connections.

\section{Hybrid Forwarding Strategies}

Hybrid forwarding schemes may combine different techniques to perform a forwarding decision. For instance, $\mathrm{Yu}$ et al. [99] proposed a hybrid routing scheme, namely Hierarchical Bloom-Filter Routing, using Bloom filter based on properties of content for advertising. In the proposed forwarding scheme, the network area is divided into geographical partitions, and each vehicle is partitioned into corresponding clusters. The Bloom filters are used to announce name prefix in each partition to achieve a lower time and less storage and communication overhead. Similarly, Deng et al. [124] proposed a hybrid forwarding strategy for location dependent and location independent information in VANETs based on NDN. This forwarding scheme addresses the effectiveness of forwarding issue in wireless environment where interest and data packets are flooded. Only one wireless interface is used in FIB and PIT tables, by using opportunistic and probabilistic forwarding strategy, which is associated with the geographic information. The authors also proposed a retransmission and acknowledgment mechanism for interest and data forwarding to achieve efficient and reliable communication.

Kaur et al. [125] proposed a forwarding method based on fuzzy membership function to improve location-based transmission, content aggregation, and distributed mobility management. This work has been extended in [126] by analyzing the existing VANET based on NDN solutions in terms of optimal value of aggregation time, time to live, and control and computation overheads. Deng et al. [127] proposed a Prior-ResponseIncentive-Mechanism for cooperative downloading of bigsized and popular content in VANETs-LTE based on NDN by taking the advantages of in-network caching and multisource into account. Prior-Response-Incentive-Mechanism is a game theoretic solution to enable the nodes to calculate optimal strategy.

Fabricio et al. [128] designed a content-centric communication mechanism for vehicular networks called InteRestCentric Mobile Ad Hoc Network for Vehicular Environments. In this mechanism, each node uses an Active Prefix and includes it in the interest packet. The node prefix is used as a node identifier, while the application interest uses it to perform a name searching and group formation. The proposed mechanism forwards the messages according to the propagation direction, the source prefix, and source position. However, this scheme does not consider the in-network caching scheme. In another work [129], the authors proposed a multihop and multipath routing algorithm for VANETs based on NDN architecture with the aim to ensure faster data retrieval and improve users' QoS and QoE. The authors defined new fields and embedded them in both interest, data packets, as well as in FIB table. Also, they developed three different techniques for path selection by distributing the traffic uniformly to all possible paths, select paths based on their latency, and finally distribute the traffic uniformly to all possible paths with the lowest latency. This scheme supports the mobility and velocity (speed) of vehicles. Further, the authors extend their work in [130] by performing the forwarding decisions in the strategy layer of NDN and develop a routing strategy based on V2V Multihop, Multipath and Multichannel communication aiming to improve the QoE for users in VANETs.

Guo et al. [132] proposed a new FIB selecting algorithm in a highway environment based on Link Expiration Time algorithm. The best outgoing interface in the FIB is selected by calculating the Link Expiration Time and the available probability based on the used distance, speed, and accelerations. Authors also defined two fields in PIT table: the receive time and tolerance time. The receive time represents the time when the first interest arrives, and the tolerance time represents the biggest time of each table entry. Similarly, Hussain et al. [133] proposed a receiver-based forwarding scheme to avoid unnecessary multiple paths. By using Signal-to-Interferenceplus-Noise Ratio and forwarding zone, a best forwarder vehicle is selected where it should be nearest to the destination. This scheme minimizes unwanted formation of multiple paths and reduces the number of hops to the destination. However, it has a limitation in excessive retransmissions, which increase the end-to-end delay. In the same way, Riu et al. [134] presented a multiple unicast paths forwarding scheme that replaces the flooding method with unicast interest forwarding paths. The authors proposed that the selection of next hop is based on FIB selecting process by using different parameters and link quality metrics. This helps to select the best path where the data can return successfully through the designated path. However, the authors did not take the mobility parameters into consideration, instead they only considered a constant motion model for vehicles.

Apart from the aforementioned schemes, IP-based forwarding is also employed for the realization of NDN-based vehicular networks. Saxena et al. [131] implemented IP-based data forwarding schemes such as Epidemic, Spray and Wait using NDN on the sparsely-connected real vehicular testbed. These NDN-based forwarding schemes study the performance of name-based forwarding for retrieving, disseminating data and it validates the performance and usability of $\mathrm{CCN}$ over VANET. However, their proposed schemes do not consider the forwarding loop and duplicate at the content level, while operating on IP-based routing mechanisms.

In a nutshell, from above-described strategies, hybrid design of forwarding scheme may provide better forwarding decision by combining various techniques. However, with such a large list of options to choose from, selecting the right set of parameters to combine and design one hybrid strategy that also meets QoS requirements of various applications, while reducing interest packets propagation overhead, is a cumbersome task.

\section{Distributed Forwarding Schemes}

The selection of interest forwarders in such schemes is totally distributed, and based on various metrics such as 
velocity of the vehicles, interest satisfaction rate, etc. For instance, Ahmed et al. [135] proposed a distributed interest forwarder selection scheme for vehicular NDN to limit the interest broadcast storm. In this scheme, each vehicle has information about its neighbor's speed, direction, and position. Vehicles use this information to select potential forwarders in the backward and forward direction which have maximum connectivity time and good link quality. While, Tiennoy et al. [139] designed a new protocol for data dissemination that guides interest packets toward the direction of the data producer. The authors modified both interest and data packets by adding new fields for geolocation information and timer in order to overcome the broadcast storm.

Similarly, Wahid et al. [136] developed a forwarding protocol for VCCN in order to mitigate the interest broadcast storm and solve the disconnected link problems. In the extension of their work [137], the authors compared their scheme with other routing schemes in VCCN in terms of end-to-end delay, packet delivery ratio, etc. Their proposed protocol has two phases: the first is intersection information sharing where each vehicle maintains its neighbor information table in order to select the best forwarder node. The second phase is interest/data packet forwarding phase where vehicles select the best forwarder based on the information shared and the speed of vehicles that matches with the consumer vehicle. Similarly, Yaqub et al. [140] presented a bio-inspired distributed interest forwarding scheme in NDN-driven VANET, where interest packets are forwarded through multiple paths toward the provider. On the other hand, Drira et al. [98] proposed an NDN query mechanism namely NDN-Query with the aim to collect and aggregate data from distributed vehicular networks using V2X communication. The data collection in NDN-Query is based on dynamic names, and is performed in two-steps: the first step is query dissemination towards the data source and the second one is about the query source response collection and aggregation.

In addition to the discussed schemes, Software-Defined Networking (SDN) has also been leveraged for the realization of NDN-based vehicular networks [156]. Soua et al. [138] investigated the role of SDN to support both CCN and floating content in order to improve content storage, its dissemination, and forwarding in VANETs. They proposed an SDN-based discovery mechanism to guarantee the QoS for each flow and to support multi-path communication for fast data retrieval. We believe that involving SDN in VANET [26] will not only improve distributed forwarding decisions, but will also help the controllers to get a global view of the network, thereby improving the routing efficacy, quality of service, and content dissemination.

\section{E. Push Traffic Forwarding Support}

NDN is a pull-based architecture where communication should be initialized by a consumer node. However, vehicular networks contain many push-based applications such as traffic information dissemination, advertisement dissemination, warnings, to name a few. To support such push-based communication, some techniques have been proposed in the literature.
Arnould et al. [141] proposed a new architecture that targets hybrid VANET based on CCN project. The authors defined a new type of unsolicited packet called Event Packet to provide vehicles the ability to disseminate emergency messages when necessary without any previous interest (push). Also, they defined a prioritization mechanism to favor the transmission of certain types of packets over others and guarantee quality of service. Similarly, Majeed et al. [142] proposed a pushbased critical data forwarding scheme for data dissemination in VNDN. The extension of this work [143] allows vehicles to broadcast an emergency message to one-hop neighbors where the producer sends one-hop beacon message that contains meta-data about new chunks. When a neighbor receives the beacon, it makes a temporary PIT entry to cache the incoming data chunks instead of treating it as unsolicited chunk and thus drops it. However, this work did not take into consideration the mobility of intermediate nodes on the reverse path, which may negatively affect network performance.

From the above discussion, it is evident that push-based strategies are good for disseminating emergency related time critical messages. However, the support of push-based traffic in NDN requires a native design without violating NDN primitives. Moreover, handling unsolicited packets require change of NDN engine. Also, allowing such kind of packets provides opportunities for Denial of Service attack on intermediate nodes. One of the promising solutions for Push-based support is the use of persistent interest packets [157].

\section{F. Prioritization Forwarding Solutions}

In prioritization forwarding schemes, traffic is classified into multiple types with varying priority value, that are used during the forwarding process to ensure QoS support. Amadeo et al. [144] proposed a prioritization mechanism for nameddata vehicular networks based on using name-prefixes that divide the vehicular data traffic into two priority categories, i.e. High and Low. The name prefixes are used to set the defer time selection locally at each vehicle. In the extension of this work [145], the authors defined a freshness value in the interest packet and a smart strategy layer to decide where the packet should be forwarded or retransmitted. Interests with low priority are forwarded only over the 802.11 Outside the Context of a Basic service set (OCB) interface, while interests with high priority are forwarded to both faces. They also proposed a timing algorithm for multi-hop delivery packets in $\mathrm{V} 2 \mathrm{~V}$ and $\mathrm{V} 2 \mathrm{R}$. However, this work assumes that global content popularity is known, which is not very easy to calculate in a distributed VANET environment. Moreover, this mechanism performs poorly under high network loads. Similarly, in [102] the authors proposed a Service-based system architecture. It defines a naming policy, service sub-layer, and service prioritization policy in order to manage information dissemination, and to allow service exchange and improve content delivery.

Boukerche et al. [146] proposed link stability-based interest forwarding for content request protocol in VNDN to mitigate interest broadcast storm. This forwarding scheme uses differ time to consider the predicted link stability between the vehicles and to determine the priority level of each neighbor. 
The main objective of preferring stable links is to ensure the selection of most suitable paths so that data packets be sent over the same paths in reverse direction on which interest packets are forwarded.

Ensuring QoS support in NDN requires design of the naming scheme, where application type (e.g., time-sensitive, comfort, traffic applications, etc.), and task class (e.g., actions to perform, data download, content sharing among vehicles, etc.) should be listed. Prioritizing forwarding based on these classes will improve the QoS. However, a careful naming convention and validation system are needed at the network level.

\section{G. Distributed Content Dissemination Schemes}

Content dissemination and rapid content access [158] are the key features of NDN, especially when coupling it with innetwork caching. Deng et al. [149] developed a simple intervehicle one-hop large-size content distribution mechanism based on NDN architecture. This mechanism allows multiple methods including multi-source supply, intelligent response, break-point resume, and adaptive switching to be fulfilled. However, this work creates a huge amount of redundant data while distributing content. Similarly, Li et al. [150] proposed two mechanisms based on NDN in VANETs, which include adaptive switching and piece-wise random walk to reduce the redundancy of data packets and to improve the efficiency of the network.

Furthermore, Talebifard et al. [147] proposed a method for content distribution in VANETs based on exploiting the benefit of multiple paths in $\mathrm{CCN}$ by using a selective randomized network coding to improve the reliability and robust distributed dissemination. The objective of using the network coding is that the source vehicles send out the data chunks and the intermediate vehicles perform a linear combination of received data chunks, and send to the next vehicles. Talebifard et al. [148] also designed a selection network coding approach for crowd sensing in the vehicular cloud in order to provide efficient and reliable information dissemination. This approach is based on the semantics of data information and the connectivity of each node within the cluster. In the same way, Bouk et al. [151] proposed a forwarding scheme that allows vehicles to send one interest in order to request multiple data from different content sources. However, the proposed solution produces more overhead.

To this end, low latency content access and rapid dissemination are considered as important metrics to evaluate network performance. Thus, to achieve a better ubiquitous content dissemination in distributed schemes, the in-network caching coupled with content properties should be taken into consideration.

\section{H. Forwarding Control Solutions}

As aforementioned, all of the NDN data structures, i.e. CS, PIT, and FIB tables are involved in NDN forwarding process. Different control mechanisms have been proposed to avoid table overflow and mitigate other attacks. Ahmed et al. [152] designed a propagation scheme for VNDN based on controlled data packets to limit data flooding by using the hop counter $h$ in interest packets and Time-To-Live (TTL) in data packets. Each vehicle in VNDN includes hop counter $h$ to keep the record of number of hop(s) that an interest packed passed through. The objective of using the TTL field is to limit the additional copies of the data packets. Similarly, Ahmed et al. [153] proposed a controlled data and interest evaluation scheme to control the data broadcast storm for VNDN. The authors defined two new fields: the first indicates the number of hops $h$ and is included in the interest packets, while the second is data dissemination limit and is embedded in data packets. The objective of using these fields is to limit the additional copies of the data/content. However, this work did not take the mobility in the reverse path into account.

Bouk et al. [154] presented a dynamic PIT entry lifetime mechanism for vehicular NDN in order to reduce the number of PIT entries, and accelerate routing and forwarding process. Dynamic PIT entry lifetime allows each relaying vehicle to calculate the PIT entry lifetime for each incoming interest based on the number of hops and the interest satisfaction rate of the vehicle itself. The PIT entry is kept active for higher value of interest satisfaction rate and hop count. However, compromised user can generate infinite number of interest with valid names, where intermediate nodes will treat them regularly, and might produce PIT overflow attack. Similarly, nodes can pollute the cache store of one-hop intermediate nodes with fake content, or even make a non-popular content popular in case of popularity-based caching. Hence, more in-depth investigation on forwarding and security issues is needed.

\section{Summary and Insights}

In this section, we provided a comprehensive overview of the existing data forwarding strategies proposed for NDNVANETs. Based on the working principles and the design purpose of existing forwarding strategies, we classified them into the following eight categories: 1) location-based forwarding, 2) neighbor-based forwarding strategies, 3) hybrid forwarding strategies, 4) distributed forwarding schemes, 5) push traffic forwarding support, 6) prioritization forwarding, 7) distributed content dissemination, and 8) forwarding control solutions. We critically overviewed all the eight categories while focusing on their pros and cons. Given the existing strategies and applications of NDN-VANETs, we believe that geo-location based forwarding along with hybrid schemes may help VANETs to overcome mobility issue and thus may provide fast content dissemination and access. Also, the support of push traffic needs a careful design on top of persistent interest without violating NDN primitives. It is also worth noting that due to the salient features of VANET, the core NDN architecture may not work well. For instance, in VANET, one node may require information from multiple nodes, such as traffic information from a defined geographical location. In such situation, traditional NDN will not work, and we need to make necessary changes to the underlying architecture so that the data structures such as PIT and FIB respond to the situation accordingly. In this regard, a tailored NDN architecture is essential for VANET. 
TABLE VII

SUMMARY OF EXISTING NDN-VANET CACHING STRATEGIES

\begin{tabular}{|c|c|c|c|c|c|c|}
\hline Ref. & Comm. Model & Scenario & Comparison & $\begin{array}{l}\text { Parameter } \\
\text { Evaluation }\end{array}$ & Simulation & Year \\
\hline \multicolumn{7}{|c|}{ Probabilistic Caching Scheme } \\
\hline [159] & - Vehicle-to-Vehicle & $\begin{array}{l}\text { - Road Segment Dissemina- } \\
\text { tions }\end{array}$ & $\begin{array}{l}\text { - AlwaysCache } \\
\text { - Probabilistic } \\
\text { Caching }\end{array}$ & $\begin{array}{l}\text { - Cache Hit Ratio } \\
\text { - Delay } \\
\text { - Average Hop Count }\end{array}$ & ndnSIM, SUMO & 2016 \\
\hline [160] & $\begin{array}{l}\text { - Vehicle-to- Infrastruc- } \\
\text { ture }\end{array}$ & - Circle/Mesh Topology & - Not mentioned & $\begin{array}{l}\text { - Content Store Size } \\
\text { - Number of Paths } \\
\text { - Success Probability } \\
\text { - Success Probability } \\
\text { Time }\end{array}$ & AMPL, ndnSIM & 2017 \\
\hline \multicolumn{7}{|c|}{ Cooperative Caching Scheme } \\
\hline [103] & $\begin{array}{l}\text { - Vehicle-to-Vehicle } \\
\text { - Vehicle-to- Infrastruc- } \\
\text { ture }\end{array}$ & $\begin{array}{l}\text { - Highway Multimedia } \\
\text { Streaming }\end{array}$ & $\begin{array}{l}\text { - } \\
\text { Caching }\end{array}$ & $\begin{array}{l}\text { - Start-up delay } \\
\text { - Playback Freezing }\end{array}$ & NS3, ndnSIM & 2014 \\
\hline [161] & $\begin{array}{l}\text { - Vehicle-to-Vehicle } \\
\text { - Vehicle-to- Infrastruc- } \\
\text { ture }\end{array}$ & - Highway Scenario & - Not mentioned & $\begin{array}{l}\text { - Playback Delay } \\
\text { - Play Buffer Utiliza- } \\
\text { tion }\end{array}$ & NS3, ndnSIM & 2015 \\
\hline \multicolumn{7}{|c|}{ Content Popularity-based Caching Scheme } \\
\hline [162] & - Vehicle-to-Vehicle & - V2V Scenario & $\begin{array}{l}\text { - Leave Copy } \\
\text { Everywhere } \\
\text { - Probabilistic } \\
\text { Caching } \\
\text { - Leave Copy Down }\end{array}$ & $\begin{array}{l}\text { - Average Time Delay } \\
\text { - Cache Hit Ratio } \\
\text { - Cache Hit Distance }\end{array}$ & SUMO & 2017 \\
\hline \multicolumn{7}{|c|}{ Hybrid Caching Scheme } \\
\hline [163] & $\begin{array}{l}\text { - Vehicle-to- Infrastruc- } \\
\text { ture }\end{array}$ & - Motorway Scenario & - Reactive Caching & $\begin{array}{l}\text { - Cache utilization } \\
\text { - One-hop Ratio } \\
\text { - Resolved Interest } \\
\text { Ratio }\end{array}$ & NS3, ndnSIM & 2016 \\
\hline [164] & $\begin{array}{l}\text { - Vehicle-to- Infrastruc- } \\
\text { ture }\end{array}$ & $\begin{array}{l}\text { - Highway Scenario } \\
\text { - Intersection Scenario }\end{array}$ & $\begin{array}{l}\text { - Leave Copy Ever- } \\
\text { where } \\
\text { - Leave Copy Down } \\
\text { - Edge Caching } \\
\text { - Consumer Cache } \\
\text { - No Cache }\end{array}$ & $\begin{array}{l}\text { - Network Delay } \\
\text { - Hop Reduction Ra- } \\
\text { tio } \\
\text { - Cache Utilization }\end{array}$ & ndnSIM, SUMO & 2018 \\
\hline
\end{tabular}

\section{NDN-VANET IN-NETWORK CACHING SCHEMES}

In principle, the Internet was designed to forward content requests to its original producer which requires nodes to be continuously connected to fulfill the consumers' demands. Thus, the current IP-based network witnesses huge load, with long content retrieval delay and bandwidth consumption. Also, if the original content producer fails to connect, it is likely to suffer from single point-failure, and the content availability will be affected. NDN proposes in-network caching, where the network itself is responsible to cache (node local decision), and serve the content requests that can reduce the overhead at the content provider side, where it is not required to be continuously connected as the network already has multiple copies of the same content that can be used to serve requests of different consumers [77], [165], [166].

From VANETs perspective, efficient caching can play a very important role to disseminate the content towards and among vehicles both quickly and in a cost-efficient manner. In this section, we survey in-detail the existing in-network caching schemes. We also classify them into various categories based on how caching technique functions. Table VII shows a summary of these discussions.

\section{A. Probabilistic Caching Schemes}

These schemes aim to provide a probabilistic content caching decision to increase the diversity of cached content in order to improve the content dissemination.

Deng et al. [159] designed a distributed probabilistic caching strategy in NDN-based VANETs. The strategy is meant for both the nodes on the forwarding path and for all nodes that received data in specific range. Nodes in distributed probabilistic caching decide whether to cache content or not through probabilistic estimation which is based on three main factors: the demand and preference of vehicles, the importance of vehicles in the geo-based network, and the relative movement of the receiver and the sender. However, the communication between nodes and the proposed packet processing to make a caching decision may affect the communication performance and increase its delay. Similarly, Mauri et al. [160] proposed a caching scheme that pre-distributes the content in V2I scenario in order to maximize the probability of average content retrieval. The authors formulated the problem 
of optimally allocating contents in the network as an Integer Linear Program. Moreover, they studied the impact of several parameters like number of users in the system, the access point available bandwidth, the propagation latency, and the caching capacity at disposal on the retrieval probability. However, this work does not benefit V2X communication especially where vehicles cannot be connected to the base station all the times, hence a distributed caching policy between vehicles, without involving RSUs or base stations, is required to enhance the content access.

Probabilistic caching may help to improve the content diversity in the network; however, the random cache decisions based on the use of probabilistic metrics may affect the content popularity, where a non popular content can be influenced by malicious users to be popular (i.e. sending a storm of content requests). Consequently, intermediate vehicles/nodes may reply to different requests via their cache stores and increase the cache hits, or even caching the content, that is in fact non-popular, and may not help in the ubiquitous content dissemination and end by increasing either cache hit in intermediate nodes, or cache a non-future requested content, which make the caching useless.

\section{B. Cooperative Caching Schemes}

Various network models have explored the cooperating caching aiming to enhance the content sharing, including ICN [167], in which the content cache process may be applied under more than one administrative authority, or autonomous system. Quan et al. [103] proposed an innovative highwaycustomized ICN-based cooperative caching solution to improve the QoE of multimedia streaming services. Also, the authors developed two novel social cooperation schemes: partner-assisted and courier-assisted for movement of vehicles in the same and opposite direction carrying interest report and data message to improve the caching efficiency for information-centric multimedia streaming [168].

Liu et al. [161] discussed caching placement issue to decide in which node, the content should be cached. The authors proposed two algorithms: the first for caching node selection that uses the minimum vertex cover set algorithm to initialize the status of nodes in VANET and solves the issue of cache initialization. The second algorithm is collaborative caching based on socialized relations to determine the caching point in order to solve the vehicle mobility problem.

Although cooperative caching schemes may result in faster content retrieving, but the current works require modifications to NDN nodes or forwarding decision, that may violate NDN primitives. Furthermore, the cooperative caching mechanisms also need to take into account, different VANET scenarios.

\section{Content Popularity Caching Schemes}

In content popularity caching schemes, the caching decision must be taken according to the popularity of the content, where the most popular and frequently used content should be cached instead of non-popular content. Zhao et al. [162] proposed a community similarity and population-based cache policy for $\mathrm{V} 2 \mathrm{~V}$ communications. This strategy is based on dynamic probability caching scheme that estimates the community similarity and privacy rating of vehicles and selects the caching vehicle based on content popularity to reduce the cache redundancy. The authors also defined a popularity prediction-based cooperative cache replacement mechanism to predict and rank popular content during a period of time and added this content popularity statistics field into the data packets.

Content popularity based caching schemes can improve the content diversity, dissemination, and reduce the content access delay by ensuring only the most used/popular content is cached in the network layer, closer to requesters. However, these schemes may suffer in case of dynamic content (that needs to be generated upon the requests), or use-once content that cannot be known whether it is popular or not.

\section{Alternative Caching Schemes}

Alternative caching solutions may use different caching decision techniques. For instance, a geo-location based caching scheme have been proposed by Grewe et al. [163]. The authors proposed a proactive caching approach for VANET based NDN that aims to improve the data delivery by distributing the content one-hop away from the consumer. This scheme uses geo-location, current velocity of the vehicle, and its direction as input parameters to demonstrate an efficient content distribution mechanism facilitating RSU-based caches. However, this scheme neither considers V2V communication nor the variable speed of vehicular nodes. Similar, Khelifi et al. [164] proposed a proactive caching-based mobility prediction scheme that uses a deep leaning module, called Long Short Time Memory, to predict the next RSU where the content will be proactively cached. The aim is to store the content in the right place and the likelihood of request for this content is high. However, the proposed solution did not take $\mathrm{V} 2 \mathrm{~V}$ communication into consideration.

\section{E. Summary and Insights}

In this section, we investigated NDN based in-network caching schemes in the context of VANET, and broadly categorized them into probabilistic caching, cooperative, content popularity, and alternative caching schemes. In summary, innetwork caching is one of the main features of ICN that aims to enhance the overall network performance by reducing content access and delivery delay. The network layer is responsible to reply to consumers' requests by caching content in the intermediate nodes. Decoupling the content from its original owner will provide a distributed content caching, where the original content owner does not have control, as the owner no longer decides who will cache its content and where. Also, the content reproducing and re-distribution will violate content copyright and user privacy, hence a careful business model that may handle these transactions is needed in parallel with caching scheme design.

\section{MOBILITy IN NDN-BAsed VANET NetworkS}

In the current IP model, every device interface is required to obtain an IP address in order to allow it to communicate with 


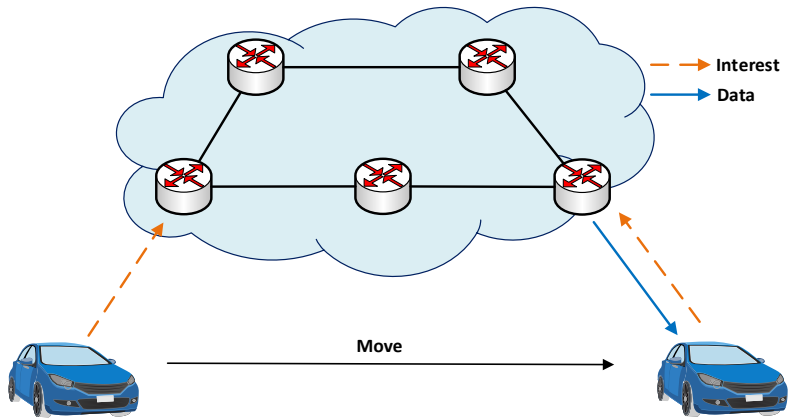

Fig. 5. NDN Mobility Support for VANET.

other entities. Mobile devices cannot communicate during their movement until they get another IP address from the newly connected network. Solutions such as MobileIP [169] and Host Identity Protocol [170] have been proposed to overcome this issue. However, these schemes do not help to solve the content mobility problem. By using content names instead of IP address in NDN [171], [172], accessing a content requires only the content name regardless of the producer host address. Hence, mobile nodes may have a better experience on accessing content during the mobility, without requiring a new address from the connected network, and may continue communicating and accessing content even if the publisher is unavailable by using in-network caching feature of the ICN.

With the basic use of NDN, especially naming and innetwork data caching, NDN may outperform TCP/IP [173] and may also help to cope with mobility and sporadic connectivity issues. NDN mobile nodes can simply re-request the missing data packet during the movement. However, other challenges arise for NDN mobility [174], especially in VANET environments where the high speed of vehicles and their movement in the different directions for intermittently short time makes the network management a challenging task. Figure 5 illustrates a simple example of vehicle movement after sending an interest, where afterwards, it should re-issue the same interest requesting the same data.

Various schemes have been proposed to address mobility challenges in VANET from NDN perspective. In the following we put light on the existing mechanisms. Wang et al. [175] proposed a routing protocol namely MobiCCN aiming to address the mobility and mobile content publishing issues. MobiCCN uses two routing protocols: the standard CCN protocol, and a greedy protocol. Benefiting from naming, the authors used two prefixes to distinguish between the routing protocol namespace ccnx:/ and greedy:/. In MobiCCN, each router and user are assigned a virtual coordinate from the underlying hyperbolic space $H$. The greedy protocol embeds the virtual coordinate into the content name of a packet. Each router maintains neighbors' coordinates table to calculate the distance between the destination and each of its neighbors and selects the closest neighbor to the destination. In case of mobility of the original data producer, the router forwards an update message to its host router and each router receives this message, updates the corresponding entries in its FIB table, then the new interests can be transmitted towards the source.
Yan et al. [85] proposed a distributed NDN mobility management scheme that uses NDN's on-path caching and embedded information about the direction of node mobility (movement) in the content name. These information are used to select the forwarding node along the same path as the vehicle's moving path, with the chance that the data has been cached on the path. This scheme manages both a single mobile node and an entire mobile subnet referred to as network mobility. However, the node movement is not always predictable and the use of content name to carry more information may affect the lookup performance and results a poor design of a unified naming scheme. In the same way, Wang et al. [176] designed a preference-aware fast interest forwarding for video streaming. Each node chooses only the nodes that have the similar mobility and video preference with it. The authors defined a new table in each node to maintain the content caching status for these nodes, nodes select next hop based on matching the requested video with an entry in the proposed table.

Duarte et al. [177] aimed the problem of reverse path partitioning that frequently degrades application performance. The authors proposed a scalable mechanism called Auxiliary Forwarding Set to address this problem and improve VNDN application regardless of receiver mobility. The auxiliary forwarding set uses several mobility factors and the NDN core by identifying an extra set of eligible nodes to forward data packets where the retransmissions are required due to reverse path partitioning. However, this work degrades the system performance in case of low vehicle densities. To achieve and explore the effects of low vehicle density in high mobility scenarios, the authors extended their work [178] and proposed two solutions namely VNDN agent delegation, and VNDN store-carry-forward. The former solution targets scenarios with infrastructure support and the latter without infrastructure support. Nevertheless, the proposed solution suffers from the broadcast storm and message redundancy problems. Also, it creates a huge amount of redundant messages leading to the collision, congestion, and overhead.

Similarly, the authors analyzed and studied the existing source mobility solutions, and evaluated the effect of NDN source mobility in [179]. Therefore, they proposed a new solution based on the concepts of floating content and home repository in order to improve the content delivery. Furthermore, authors extended their previous solution in [180], and proposed a framework named MobiVNDN in order to improve VANET applications performance in the high mobility scenarios, overcome the broadcast storms, and message redundancy. Wang et al. [181] introduced fog computing into information-centric Internet of Vehicles in order to provide mobility support and avoid the incessant FIB update. The authors used fog computing paradigm to perform computation, storage, and location-aware, and taking the data characteristics into account to design a mobility support scheme for Internet of Vehicles service. However, more investigation is needed to argue on the feasibility of applying fog computing on top of NDN-VANET. 


\section{A. Summary and Insights}

This section outlined NDN based VANET mobility solutions, where VANETs are considered as dynamic and high mobility environment where the prediction of vehicle's movement is not an easy task. Bringing NDN to VANET may solve some of the mobility related issues. Meanwhile, vehicles may act as content consumers, providers, and intermediate nodes that cache the content to improve the network performance. The mobility of each entity has different impact on the network, especially providers mobility, where more in-depth investigation is needed. Similarly, ignoring intermediate nodes mobility is like using NDN without in-network caching. Furthermore, a careful management solution for hand-off mobility is needed for consumer mobility, where re-issuing interest during the mobility may not be a suitable solution. Finally, treating the mobility at the forwarding level is promising to solve most VANET-NDN issues, for both consumer and producer movements.

\section{NDN-VANET SECURITY AND PRIVACY SOlUtions}

Current Internet security is based on creating a secure channel between end-users to secure the content transmission among them. However, this principle may have several drawbacks such as: the secure information exchanged cannot be used again if the session expires. In contrast, NDN provides security to the content itself by embedding cryptographic signature and some auxiliary related information in every data packet [182]. By using content-based security, NDN solves many problems that exist in IP-based networking [183]. For example, Distributed Denial of Service (DDoS) attacks reap benefits from knowing the IP address, but NDN reduces the effectiveness of DoS attacks because a node is not directly addressable. However, other security and privacy challenges still exist in NDN when used with VANET [47]. In this section, we highlight the security and privacy solutions in the sphere of NDN-based VANET.

\section{A. Security Solutions}

The term security may refer to securing all network infrastructure including devices, producer data, and communication from inside and outside attacks. Signorello et al. [23] surveyed various security challenges existing in NDN-driven VANETs, such as interest flooding, cache poisoning, and other privacy issues. However, a lack of existing security solutions and technical discussing were missing. Rezaeifar et al. [184] proposed a trust-based cache poisoning attack mitigation technique in NDN. The authors took into account the trust, credibility, and feedback into account to identify invalid contents. Based on these metrics, a trust evaluation mechanism is established that decides on the validity of the content. Although this scheme, in essence, is not meant for VANET; however, with necessary tweaks, it can be used for mobile vehicular networks.

Wang et al. [185] designed a secure mechanism for data collection from mobile vehicles, which allows manufacturers to verify the integrity and authenticity of incoming content and to protect the privacy of mobile users from malicious attacks and nodes. The content in this mechanism is encrypted with the public key of the database server and tagged with its publisher's signature. The authors also assumed that the data collector has access to mobile's public key. This is reasonable for manufacturers to record and store the public key of database server inside vehicles before release. From in-network cache perspective, Khelifi et al. [186] introduced a reputation-based blockchain scheme in order to secure the cache store and overcome the missing trust between cache stores and consumer vehicles. The proposed scheme caches only the trusted data based on using blockchain network and assigning each cache store a reputation value, that is increased/decreased based on the served content.

\section{B. Privacy Solutions}

Preserving the content and user privacy in any network including VANET is essential and of paramount importance for user satisfaction. Chowdhury et al. [101] designed a trust model for autonomous vehicular applications based on NDN, named AutoNDN, to prevent the false data and vehicle tracking. Authors proposed a four-level hierarchical trust model including autonomous-Vehicle organizations, manufacturers, Vehicles, and data, then they proposed a naming scheme to detect false information. Furthermore, they also addressed vehicle tracking by using a pseudonym and proxy-based scheme to make it more secure and difficult for attackers to track vehicles for such information. Moreover, AutoNDN application has been implemented on top of Raspberry Pi-based mini cars in a wireless environment. Similarly, these authors proposed an anonymous pseudonym-renewal and pseudonymous authentication scheme for NDN-driven VANET [187]. Pseudonyms are used for vehicles as node IDs instead of real IDs to prevent tracking of the vehicle. The pseudonym consists of two components: the vehicle pseudonym and manufacturer pseudonym. Also, a preliminary version of anonymous pseudonym renewal method has been defined that uses a certificate issuing proxy.

\section{Summary and Insights}

As the research community takes the security and privacy from a global view of wide NDN network, without focusing on VANET domain-specific applications. The security and privacy concerns still need more efforts and investigation. However, as VANETs have unique characteristics such as mobility patterns, intermittent communications, applications, than the normal network, designing specific solutions for VANET is required to enforce security, privacy, and trust relationship. Furthermore, access control, trust management, anonymity, and copyright preserving need to be further investigated to coexist with NDN.

\section{Standards, Solutions, and Simulation Tools}

NDN is still in its early phases of development and deployment. Various efforts on standardization and developing commercial solutions have been presented in the literature as well as simulation tools to facilitate the development and testing such applications. In the following, we present an overview of the existing standards, solutions, and simulation tools. 
TABLE VIII

SUMMARY OF EXISTING NDN-VANET SIMULATION TOOLS

\begin{tabular}{|c|c|c|c|c|}
\hline Simulators & Source & Pros & Cons & Recommendation \\
\hline ndnSIM & $\begin{array}{l}{[78],[82],[88],} \\
{[93],[97],[98],} \\
{[103],[115],[116],} \\
{[118],[120],[127],} \\
{[129],[130],[132],} \\
{[134],[139],[142],} \\
{[143],[149],[150],} \\
{[159]-[161],[163],} \\
{[164],[181],[186]}\end{array}$ & $\begin{array}{l}\text { - Open source package. } \\
\text { - Supports Linux and Mac OS plat- } \\
\text { forms. } \\
\text { - Maintains all the basic of NDN pro- } \\
\text { tocol operations. } \\
\text { - Ability to use all modules, compo- } \\
\text { nents, and NetDevice of NS3. } \\
\text { - Allows researchers to share and } \\
\text { use traffic traces and packets analysis } \\
\text { among CCNx and ndnSIM. } \\
\text { - Facilitates network-layer experimen- } \\
\text { tations by use routing, caching, for- } \\
\text { warding, and congestion management. } \\
\text { - Can run on top of the link-layer, } \\
\text { network-layer,and transport-layer proto- } \\
\text { cols. } \\
\text { - Supports large-scale experiments. }\end{array}$ & $\begin{array}{l}\text { - Limited support on the Windows plat- } \\
\text { form. } \\
\text { - Not support simulation network to } \\
\text { connect with NFD and ndn-cxx. } \\
\text { - Memory consumption by simulating } \\
\text { large-scale experiments. } \\
\text { - Missing of full backward compatibil- } \\
\text { ity of new releases. }\end{array}$ & $\begin{array}{l}\text { - V2X scenarios. } \\
\text { - Info-Traffic applica- } \\
\text { tion. } \\
\text { - Broadcasting Applica- } \\
\text { tion. } \\
\text { - Mobility scenario. } \\
\text { - Video streaming. } \\
\text { - Network Convergence. } \\
\text { - Caching Effectiveness. }\end{array}$ \\
\hline $\mathrm{NS} 2 / 3$ & $\begin{array}{l}{[83],[84], \quad[86],} \\
{[87],[91], \quad[94],} \\
{[117],[121],[124],} \\
{[135]-[137],[140],} \\
{[141],[152]-[154],} \\
{[176]}\end{array}$ & $\begin{array}{l}\text { - Open source package. } \\
\text { - Supports Linux, Mac OS, and Win- } \\
\text { dows platforms. } \\
\text { - Create topologies with full TCP/IP } \\
\text { protocol stack. } \\
\text { - Supports large-scale experiments. }\end{array}$ & $\begin{array}{l}\text { - Network simulation scalability in } \\
\text { large-scale topologies (limited memory } \\
\text { and computation time). } \\
\text { - Not supports the basic of NDN pro- } \\
\text { tocol operations. } \\
\text { - Created manually own methods and } \\
\text { functions for NDN. }\end{array}$ & $\begin{array}{l}\text { - Traffic Ticketing. } \\
\text { - Info-Traffic applica- } \\
\text { tion. } \\
\text { - V2X scenarios. } \\
\text { - Broadcasting Applica- } \\
\text { tion. }\end{array}$ \\
\hline $\begin{array}{l}\text { Other (OM- } \\
\text { NET++, } \\
\text { QualNet, } \\
\text { C++, } \\
\text { Matlab, } \\
\text {.. ) }\end{array}$ & 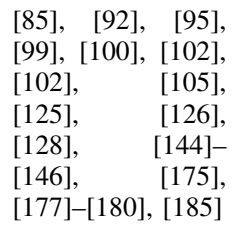 & $\begin{array}{l}\text { - Open source package. } \\
\text { - Supports Linux, Mac OS, and Win- } \\
\text { dows platforms. } \\
\text { - Easily to use. }\end{array}$ & $\begin{array}{l}\text { - Not supports the basic of NDN pro- } \\
\text { tocol operations. } \\
\text { - Created manually own methods and } \\
\text { functions for NDN. }\end{array}$ & $\begin{array}{l}\text { - Info-Traffic applica- } \\
\text { tion. } \\
\text { - Mobility scenario. }\end{array}$ \\
\hline Real Testbed & $\begin{array}{l}\text { [89], [101], [119], } \\
{[131]}\end{array}$ & - Credibility and validation. & - Costly in terms of resources. & $\begin{array}{l}\text { - V2X scenarios. } \\
\text { - Broadcasting Applica- } \\
\text { tion. } \\
\text { - Info-Traffic applica- } \\
\text { tion. } \\
\text { - Mobility scenario. } \\
\text { - Caching Effectiveness. }\end{array}$ \\
\hline $\begin{array}{l}\text { Not } \\
\text { mentioned }\end{array}$ & $\begin{array}{l}{[79]-[81], \quad[90],} \\
{[100],[122],[123],} \\
{[147],[148],[162]}\end{array}$ & & - No simulation are mentioned. & \\
\hline
\end{tabular}

\section{A. Standards and Solutions}

Currently, IETF is focusing on experimental standards for NDN. These standards are an improved version of the original PARC CCN Project. In fact, NDN project is a fork version of CCN, launched in 2010 by NSF FIA project. NDN version 0.1 that has been released in August 2013 is a fork of $\mathrm{CCNx}$ version 0.7.2. In [188], the authors presented the fundamental concept for $\mathrm{CCNx}$ architecture and described the protocol which is based on the exchange of interest and content objects. In the same draft, authors specified packet fields (mandatory and optional) as well as their interpretation. Continuing on standards efforts, another work in [189] specifies CCNx messages encoding in Type-Length-Value (TLV) packet format. The draft describes different TLV types used for different elements and their encoding values. Both aforementioned drafts [188], [189] contribute directly to the deployment and adoption of CCNx architecture.
The IETF version has implementations on fd.io (Linux Foundation) [190] and CCNLite [191]. The fd.io implementation is called Community ICN (CICN) that focuses on CCNx implementation based on the specification described in [188], [189] including packet processing and socket APIs. The project also provides a set of Android/iOS applications that can be used by the researchers to demonstrate the concept and run experiments. Whereas $\mathrm{CCN}$-lite is a lightweight implementation of $\mathrm{CCN}$ architecture that can run on resourceconstrained devices, and for commercial products. CCN-lite has a tiny code and runs on multiple platforms including Unixbased operating system, Android, Arduino, and Docker, and facilitates the deployment of commercial solutions.

ICN2020 Project [192] aims to develop innovative applications (e.g., video delivery, interactive videos, and social networks) by exploiting the benefits of ICN. It also enables the integration of ICN with different technologies including 
IoT, Cloud, Content Delivery Network, and virtualization services. The project also provides a road-map to build both local and global test-beds in order to test and experiment different applications and services. Finally, it contributes to the development of common APIs and standards.

Although these projects focus on $\mathrm{CCN}$ architecture, there is a minor difference between $\mathrm{CCN}$ and NDN, including the use of partial name matching, TLV format, and Nonce in interest packets. While various converged efforts have been shown by IRTF [193]. We believe that NDN is still in its earlier adaptation phase, more standardized efforts and commercial solutions may show the real performance of ICN in general and NDN in specific, against IP-based network.

\section{B. Simulation Tools}

The research community developed many simulation tools and models that can be used for NDN-based VANETs in order to assess and address the issue of defining an accurate mobility model and to give a realistic description of vehicular traffic. Most famous simulators include ndnSim [194], ccnSim [195], NS2/3 [196], and Icarus [197] that are used for NDN, and NDN-based VANET. Many other works used different tools and languages such as C++, Matlab, OMNeT++ [198], Qualnet [199] as well as presented a real test-bed by using Raspberry Pi. From Table VIII, it can be observed that ndnSim is the most explored simulator for NDN-based VANET due to its features space, ease of use, and openness. It is to note that ccnSim and Icarus have not been used in any solution that we have covered in this survey. The reason for mentioning these tools is to help the research community to know and be familiar with all existing simulation tools and use them according to their needs.

NS-3 is an open-source network simulation platform that is implemented in the $\mathrm{C}++$ language, and is created from scratch in order to replace and overcome different issues faced in its previous version (NS-2). NS-3 allows developers to create their topologies and scenarios easily with the custom node, a full TCP/IP protocol stack is supported with different modules and extra add-ons. Moreover, trace and data analysis with visualization of the simulation is also possible. OMNeT++ (or Objective Modular Network Testbed in C++) [198] is an open source modular simulator based on $\mathrm{C}++$ language, and it is used for simulating the communication networks or other distributed systems. Also, OMNeT++ supports Linux, Mac OS/X, and Windows platforms. QualNet [199] is another network simulation software used for creating and running network scenarios, designing protocols, as well as analyzing and studying the network performance with visualizing the simulation. QualNet can be used in both Linux or Windows platforms.

ndnSim [194] is an NS3-based simulator that can implement and simulate the NDN architecture using various link-layer protocol such as point-to-point, Carrier Sense Multiple Access (CSMA), wireless, as well as different network layer protocols (IPv4, IPv6), and transport layer (TCP, UDP). ndnSim combines NDN Forwarding Daemon (NFD) and ndn-cxx codebases in order to provide a level of interoperability between simulation and prototyping. However, this integration causes many challenges, where ndnSim cannot support the connection of the simulation network with NFD and ndn-cxx in case of running on an external host. Also, every change in NFD or ndn-cxx needs to be manually integrated with ndnSIM. Other challenges faced by ndnSim include the limitation of memory consumption by simulating large-scale experiments on top of devices that have limited hardware resources. Furthermore, the absence of full backward compatibility of new releases is another problem with ndnSim.

ccnSim [195] is a scalable chunk-level simulator based on $\mathrm{C}++$ programming language that can work with Omnet++. conSim can be used to analyze and study the caching performance of large-scale scenarios in CCN network. Finally Icarus [197] is a Python-based caching simulator, which is used to implement and evaluate different ICN caching and replacement schemes.

Focusing on content-oriented networks, most researchers prefer ndnSim due to the fact that the NDN core is already implemented and respected. Researchers start implementing their algorithms and schemes without working on the NDN primitives. The use of Matlab, OMNeT++, and Qualnet may provide results, but are still not reliable as the NDN primitives and working principles are not implemented or even respected. Researcher may program the NDN primitives during the simulation, but still not yet reliable and other researchers cannot end by comparing its performance as the simulation environment is not the same.

\section{Issues And Future Research Directions}

NDN is still in its infancy and taking shape to be deployed as future Internet architecture. In the following, we present issues and challenges with current reviewed solutions, and identify future research directions for NDN-based VANETs that serve as guidelines for the research community.

\section{A. Naming}

Content naming is the main element in NDN. The current choices include hierarchical, flat and attribute-value names. Table $\mathrm{V}$ indicates that hierarchical and hybrid naming schemes attracted attention from researchers in comparison to flat and attribute-based names. This is due to the simplicity and the aggregation feature provided by hierarchical names, which may support a better scalability. On the other hand, hybrid names enhance the benefits by combining more schemes, provide an efficient and scalable lookup, aggregation rules, optimized lookup with less-memory usage, and privacy and security support. However, naming in NDN is still subject to more investigation to be tailored for VANET. One of the open challenges in the naming is the conventions and agreements on which naming scheme should be based. Users are concerned only about content, regardless of the naming of the lookup process. Currently, long variable unbounded names are used that may consume more memory, effect the FIB update process thereby adversely affecting the network scalability, especially with the high mobility constraints in VANET. A careful naming design is needed that takes the name length 
into consideration, like encoding names into small integers and use them in the forwarding and look-up process.

\section{B. Content Discovery and Delivery}

As discussed already, NDN decouples the routing and forwarding planes. Researchers focus on NDN forwarding schemes, while NDN routing plane still lacks from Internetscale algorithms that may map the existing algorithms to NDN. Furthermore, NDN does not have a dedicated transport layer. All transport functionalities are shifted into the forwarding plane. Most of VANET-based NDN forwarding schemes are based on geographical or location-based techniques. Solutions may explore some features such neighbor status, try to make the forwarding QoS supporting and distributed during the content discovery. However, choosing the next-hop must take numerous features into consideration such as: load balancing, congestion avoidance, link failure, detect and security attacks. These techniques still lack in NDN forwarding plane and need to be addressed to achieve better content discovery and delivery experiences.

\section{In-Network Caching}

The use of in-network caching helps the network to achieve better performances comparing to traditional IP networks. Several caching strategies are presented in VANET targeting the content popularity and other probabilistic solutions to keep only the popular content closer to consumers. Researchers are focusing on probabilistic and content popularity based solutions; however, due to the diversity of traffic in VANET, selecting which traffic should be cached is still an open issue. Also, as far as resources are used from nodes to the benefits of other nodes, routers and vehicles have limited cache space because of the cost and to maintain the node performance. Hence, a well-designed caching replacement strategy needs to be in place, taking into account the high mobility of vehicles, their properties and embedded sensors. Finally, proposing a complete caching management system that can deal with, not only for VANET, but also the whole Internet scale, is still among current open issues for researchers.

\section{Mobility}

A vehicular network is considered as a high mobility environment. To cope with the problems caused by mobility, most of the existing NDN mobility solutions reap benefits of innetwork caching (to enhance data availability), and vehicular destination prediction (to proactively deliver the content to the next destination), overlooking the issue of replica-node mobility which is as important as content provider mobility in VANET. Software-Defined Network (SDN) can be deployed on top of VANET to provide a global network vision about vehicles and their mobility. Similarly, mobile edge computing can be merged with NDN in-network caching to provide a wide ubiquitous and distributed cached content in the network. This will drastically enhance the content access/delivery during vehicles mobility. Moreover, applying deep learning and reinforcement learning approaches in vehicle mobility and the most frequently used content, can enhance the data delivery process [200]. Furthermore, ensuring a communication with high performance under wireless communication medium requires on-demand and advertised content retrieval support, with a careful study on the negativity effects of mobility.

\section{E. Quality of Service}

QoS support such as low latency support with better scalability and better reliability is essential for VANET applications. Researchers are currently focusing on traffic prioritization in the forwarding plane. However, designing QoS-aware protocol on top of NDN is essential and imperative, especially that NDN content delivery follows the same path in reverse as the interest. Thus, the QoS support must take the data delivery into consideration when forwarding the content discovery request. Link quality estimation, QoS provisioning, and route reservation are typical solutions to achieve such goal, especially within the unpredictable cars mobility.

\section{F. Security and Privacy}

In essence, VANET security and privacy may not be different from other applications' security. NDN follows contentbased security where security-related information are embedded in the content (usually data packets), and uses the content name to discover, forward, and deliver the content. Hence, various security and privacy issues still remain by exploring the content name, tracing the requested content, and poisoning it. The use of public key infrastructure in VANET is challenging, especially to maintain key management under the high mobility scenarios, such as keys creation, distribution, and revocation. Further, content access-control, authentication, and cached authorization are still open challenges in NDNdriven VANET environment. More investigation is needed in this direction. Furthermore, the need for VANET-based trust model that may improve content and vehicular privacy, improve infrastructure security, and avoid different attacks is always strongly desirable. Another important direction in NDN-based VANET is the performance of NDN security solutions. For instance, when a requested content is retrieved, it is important to decide whether every node along the path should verify the content, or only designated nodes must verify the content. This scenario is most important in frequently exchanged messages such as beacons. More investigation is needed in this direction to address the performance of security solutions in NDN-based VANET as well.

\section{CONCLUSION}

Named Data Networking (NDN) has emerged as one of the most widely adapted implementations of content-centric networking. The issues with current IP-based Internet (such as scalability, mobility, addressing, security, and privacy) advocate for new Internet infrastructure where the focus is on the data/content itself rather than the origin of the data/content. To this end, NDN has played a very important role in helping the realization of content-centric Internet. NDN aids plethora of application domains ranging from sensor networks to Internet 
of Things and mobile networks. Without loss of generality, in this paper, we focused on vehicular networks from the contentcentric networking perspective. More precisely, we discussed and presented the role of NDN in the realization of VANET in detail from the standpoint of existing literature, solutions, and future research and implementation issues. We first overviewed VANET applications along with their characteristics, elaborated various issues and challenges that affect the vehicular communications, then we provided a detailed overview of NDN and its architecture with the mapping between them and also discussed NDN suitable features. Through this survey, we have also presented state-of-the-art literature regarding the existing solutions, architectures and platforms, as well as every aspect of NDN such as naming schemes, content discovery and delivery, in-network caching, mobility, and security and privacy in vehicular networks, and the existing simulation tools. In the end, we also presented and identified research gaps, and challenges that may be considered by the research community to design concrete architecture for NDN-based VANET. The future of NDN in the commercialization of VANET is speculative, and we believe that more research is needed to find the common ground for applying NDN to VANET.

\section{ACKNOWLEDGMENTS}

We would like to thank the anonymous reviewers for their constructive comments and suggestions which helped us to further improve the quality, content, and presentation of this paper.

\section{REFERENCES}

[1] S. Al-Sultan, M. M. Al-Doori, A. H. Al-Bayatti, and H. Zedan, "A comprehensive survey on vehicular ad hoc network," Journal of network and computer applications, vol. 37, pp. 380-392, 2014.

[2] A. Intl, "Standard specification for telecommunications and information exchange between roadside and vehicle systems-5 GHz band Dedicated Short Range Communications (DSRC)," Medium Access Control and Physical Layer specifications, E2213-03, 2003.

[3] E. Ahmed and H. Gharavi, "Cooperative vehicular networking: A survey," IEEE Transactions on Intelligent Transportation Systems, vol. 19, no. 3, pp. 996-1014, 2018.

[4] "National Research Foundation (NSF)," [Online] http://www.nets-fia. net/, Accessed: 2018-09-17.

[5] L. Zhang, A. Afanasyev, J. Burke, V. Jacobson, P. Crowley, C. Papadopoulos, L. Wang, and B. Zhang, "Named data networking," ACM SIGCOMM Computer Communication Review, vol. 44, no. 3, pp. 6673, 2014.

[6] I. Seskar, K. Nagaraja, S. Nelson, and D. Raychaudhuri, "Mobilityfirst: Future Internet Architecture Project," in Asian Internet Engineering Conference. ACM, 2011.

[7] T. Anderson, K. Birman, R. Broberg, M. Caesar, D. Comer, C. Cotton, M. J. Freedman, A. Haeberlen, Z. G. Ives, and A. Krishnamurthy, "The nebula future internet architecture," in The Future Internet Assembly. Springer, 2013, pp. 16-26.

[8] A. Anand, F. Dogar, D. Han, B. Li, H. Lim, M. Machado, W. Wu, A. Akella, D. G. Andersen, and J. W. Byers, "XIA: An architecture for an evolvable and trustworthy Internet," in ACM Workshop on Hot Topics in Networks. ACM, 2011, p. 2.

[9] T. Wolf, J. Griffioen, K. L. Calvert, R. Dutta, G. N. Rouskas, I. Baldin, and A. Nagurney, "ChoiceNet: toward an economy plane for the Internet," ACM SIGCOMM Computer Communication Review, vol. 44, no. 3, pp. 58-65, 2014.

[10] "European Commission," [Online] https://ec.europa.eu/commission/ index_en, Accessed: 2018-09-17.
[11] T. Koponen, M. Chawla, B.-G. Chun, A. Ermolinskiy, K. H. Kim, S. Shenker, and I. Stoica, "A Data-oriented (and Beyond) Network Architecture," ACM SIGCOMM Computer Communication Review, vol. 37, no. 4, pp. 181-192, 2007.

[12] G. García, A. Beben, F. J. Ramón, A. Maeso, I. Psaras, G. Pavlou, N. Wang, J. Śliwiński, S. Spirou, and S. Soursos, "COMET: Content mediator architecture for content-aware networks," in Future Network \& Mobile Summit (FutureNetw). IEEE, 2011, pp. 1-8.

[13] N. B. Melazzi, S. Salsano, A. Detti, G. Tropea, L. Chiariglione, A. Difino et al., "Publish/subscribe over information centric networks: A Standardized approach in CONVERGENCE," in Future Network Mobile Summit, 2012, pp. 1-8.

[14] M. Brunner, "Scalable \& Adaptive Internet solutions (SAIL)," Future Internet Assembly, 2010.

[15] N. Fotiou, P. Nikander, D. Trossen, and G. C. Polyzos, "Developing Information Networking Further: From PSIRP to PURSUIT," in Conference on Broadband Communications, Networks, and Systems. Springer Berlin Heidelberg, 2010.

[16] G. Xylomenos, C. N. Ververidis, V. A. Siris, N. Fotiou, C. Tsilopoulos, X. Vasilakos, K. V. Katsaros, and G. C. Polyzos, "A survey of information-centric networking research," IEEE Communications Surveys \& Tutorials, vol. 16, no. 2, pp. 1024-1049, 2014.

[17] D. P. Arjunwadkar, "Introduction of NDN with Comparison to Current Internet Architecture based on TCP/IP," International Journal of Computer Applications, vol. 105, no. 5, pp. 31-35, 2014.

[18] V. Jacobson, D. K. Smetters, J. D. Thornton, M. Plass, N. Briggs, and R. Braynard, "Networking named content," Communications of the ACM, vol. 55, no. 1, pp. 117-124, 2012.

[19] S. H. Bouk, S. H. Ahmed, D. Kim, and H. Song, "Named-datanetworking-based ITS for smart cities," IEEE Communications Magazine, vol. 55, no. 1, pp. 105-111, 2017.

[20] M. Amadeo, C. Campolo, and A. Molinaro, "Information-centric networking for connected vehicles: a survey and future perspectives," IEEE Communications Magazine, vol. 54, no. 2, pp. 98-104, 2016.

[21] S. H. Bouk, S. H. Ahmed, and D. Kim, "Vehicular content centric network (VCCN): a survey and research challenges," in Annual ACM Symposium on Applied Computing, ser. SAC '15. ACM, 2015, pp. 695-700.

[22] Z. Zhu, J. Loo, Y. Chen, K. K. Chai, and T. Zhang, "Recent advances in connected vehicles via information-centric networking," IET Conference Proceedings, pp. 10 (8 .)-10 (8 .)(1), 2016.

[23] S. Signorello, M. R. Palattella, and L. A. Grieco, "Security Challenges in Future NDN-Enabled VANETs," in IEEE Trustcom/BigDataSE/ISPA. IEEE, 2016, pp. 1771-1775.

[24] M. A. Yaqub, S. H. Ahmed, S. H. Bouk, and D. Kim, "Interest forwarding in vehicular information centric networks: a survey," in Proceedings of the 31st Annual ACM Symposium on Applied Computing. ACM, 2016, pp. 724-729.

[25] I. Shemsi and P. Kadam, "Named Data Networking in VANET: A Survey," International Journal of Scientific Engineering and Science, vol. 1, no. 11, pp. 45-49, 2017.

[26] S. H. Ahmed, S. H. Bouk, D. Kim, D. B. Rawat, and H. Song, "Named data networking for software defined vehicular networks," IEEE Communications Magazine, vol. 55, no. 8, pp. 60-66, 2017.

[27] F. Cunha, L. Villas, A. Boukerche, G. Maia, A. Viana, R. A. Mini, and A. A. Loureiro, "Data communication in VANETs: Protocols, applications and challenges," Ad Hoc Networks, vol. 44, pp. 90-103, 2016.

[28] J. B. Kenney, "Dedicated Short-Range Communications (DSRC) Standards in the United States," Proceedings of the IEEE, vol. 99, no. 7, 2011.

[29] Y. Y. Nasrallah, I. Al-Anbagi, and H. T. Mouftah, "Enhanced Algorithms for the IEEE 802.11p Deployment in Vehicular Ad Hoc Networks," in IEEE Vehicular Technology Conference (VTC-Fall). IEEE, 2017, pp. 1-5.

[30] R. Hussain, Z. Rezaeifar, and H. Oh, "A Paradigm Shift from Vehicular Ad Hoc Networks to VANET-Based Clouds," Wireless Personal Communications, vol. 83, no. 2, pp. 1131-1158, 2015.

[31] R. Hussain, Z. Rezaeifar, Y.-H. Lee, and H. Oh, "Secure and privacyaware traffic information as a service in VANET-based clouds," Pervasive and Mobile Computing, vol. 24, pp. 194 - 209, 2015.

[32] R. Hussain, F. Abbas, J. Son, S. Kim, and H. Oh, "Using public buses as mobile gateways in vehicular clouds," in IEEE International Conference on Consumer Electronics (ICCE). IEEE, 2014, pp. 175176. 
[33] R. Hussain, D. Kim, M. Nogueira, J. Son, A. Tokuta, and H. Oh, "A New Privacy-Aware Mutual Authentication Mechanism for Chargingon-the-Move in Online Electric Vehicles," in International Conference on Mobile Ad-hoc and Sensor Networks (MSN). IEEE, 2015, pp. $108-115$.

[34] Z. Rezaeifar, R. Hussain, S. Kim, and H. Oh, "A New Privacy Aware Payment Scheme for Wireless Charging of Electric Vehicles," Wireless Personal Communications, vol. 92, no. 3, pp. 1011-1028, 2017.

[35] R. Hussain, J. Son, D. Kim, M. Nogueira, H. Oh, A. O. Tokuta, and J. Seo, "PBF: A New Privacy-Aware Billing Framework for Online Electric Vehicles with Bidirectional Auditability," Wireless Communications and Mobile Computing, vol. 2017, p. 17, 2017.

[36] Y. Zhang, S. Gjessing, H. Liu, H. Ning, L. T. Yang, and M. Guizani, "Securing vehicle-to-grid communications in the smart grid," IEEE Wireless Communications, vol. 20, no. 6, pp. 66-73, 2013.

[37] M. A. Masrur, A. G. Skowronska, J. Hancock, S. W. Kolhoff, D. Z McGrew, J. C. Vandiver, and J. Gatherer, "Military-Based Vehicleto-Grid and Vehicle-to-Vehicle Microgrid: System Architecture and Implementation," IEEE Transactions on Transportation Electrification, vol. 4, no. 1, pp. 157-171, 2018.

[38] S. A. A. Shah, E. Ahmed, M. Imran, and S. Zeadally, "5G for vehicular communications," IEEE Communications Magazine, vol. 56, no. 1, pp. $111-117,2018$.

[39] M. Laroui, A. Sellami, B. Nour, H. Moungla, H. Afifi, and S. BoukliHacéne, "Driving Path Stability in VANETs," in IEEE Global Communications Conference (GLOBECOM). IEEE, 2018, pp. 1-6.

[40] D. Kim, Y. Velasco, W. Wang, R. N. Uma, R. Hussain, and S. Lee, "A New Comprehensive RSU Installation Strategy for Cost-Efficient VANET Deployment," IEEE Transactions on Vehicular Technology, vol. 66, no. 5, pp. 4200-4211, May 2017.

[41] R. Hussain, Z. Rezaeifar, J. Son, M. Z. A. Bhuiyan, S. Kim, and H. Oh, "PB-MII: replacing static RSUs with public buses-based mobile intermediary infrastructure in urban VANET-based clouds," Cluster Computing, vol. 20, no. 3, pp. 2231-2252, Sep 2017.

[42] L. Yao, J. Wang, X. Wang, A. Chen, and Y. Wang, "V2X Routing in a VANET Based on the Hidden Markov Model," IEEE Transactions on Intelligent Transportation Systems, vol. 19, no. 3, pp. 889-899, 2018.

[43] M. Balfaqih, M. Ismail, R. Nordin, and Z. A. Balfaqih, "802.21Assisted Distributed Mobility Management Solution in Vehicular Networks," IEEE Access, vol. 5, pp. 9518-9532, 2017.

[44] M. Boban, R. Meireles, J. Barros, O. Tonguz, and P. Steenkiste, "Exploiting the height of vehicles in vehicular communication," in IEEE Vehicular Networking Conference (VNC). IEEE, 2011, pp. $163-$ 170.

[45] M. Boban, R. Meireles, J. Barros, P. Steenkiste, and O. K. Tonguz, "TVR - Tall Vehicle Relaying in Vehicular Networks," IEEE Transactions on Mobile Computing, vol. 13, no. 5, pp. 1118-1131, 2014.

[46] M. N. Mejri, J. Ben-Othman, and M. Hamdi, "Survey on VANET security challenges and possible cryptographic solutions," Vehicular Communications, vol. 1, no. 2, pp. 53-66, 2014.

[47] C. Bernardini, M. R. Asghar, and B. Crispo, "Security and privacy in vehicular communications: Challenges and opportunities," Vehicular Communications, vol. 10, pp. 13 - 28, 2017.

[48] H. Hasrouny, A. E. Samhat, C. Bassil, and A. Laouiti, "VANet security challenges and solutions: A survey," Vehicular Communications, vol. 7, pp. 7-20, 2017.

[49] F. Sakiz and S. Sen, "A survey of attacks and detection mechanisms on intelligent transportation systems: VANETs and IoV," Ad Hoc Networks, vol. 61, pp. 33-50, 2017.

[50] S. S. Manvi and S. Tangade, "A survey on authentication schemes in VANETs for secured communication," Vehicular Communications, vol. 9, pp. 19-30, 2017

[51] T. N. D. Pham and C. K. Yeo, "Adaptive trust and privacy management framework for vehicular networks," Vehicular Communications, vol. 13, pp. 1-12, 2018.

[52] S. Sharma and A. Kaul, "A survey on Intrusion Detection Systems and Honeypot based proactive security mechanisms in VANETs and VANET Cloud," Vehicular Communications, vol. 12, pp. 138 - 164, 2018.

[53] N. Gupta, A. Prakash, and R. Tripathi, "Medium access control protocols for safety applications in Vehicular Ad-Hoc Network: A classification and comprehensive survey," Vehicular Communications, vol. 2, no. 4, pp. 223-237, 2015.

[54] S. Ucar, S. C. Ergen, and O. Ozkasap, "Multihop-cluster-based IEEE $802.11 \mathrm{p}$ and LTE hybrid architecture for VANET safety message dissemination," IEEE Transactions on Vehicular Technology, vol. 65, no. 4 , pp. 2621-2636, 2016.
[55] H. Nguyen-Minh, A. Benslimane, and D.-J. Deng, "Reliable broadcasting using polling scheme based receiver for safety applications in vehicular networks," Vehicular Communications, vol. 4, pp. 1-14, 2016.

[56] S. Ucar, S. C. Ergen, and O. Ozkasap, "Multihop-Cluster-Based IEEE 802.11p and LTE Hybrid Architecture for VANET Safety Message Dissemination," IEEE Transactions on Vehicular Technology, vol. 65 , no. 4, pp. 2621-2636, 2016

[57] S. Ucar, B. Turan, S. C. Ergen, O. Ozkasap, and M. Ergen, "Dimming support for visible light communication in intelligent transportation and traffic system," in IEEE/IFIP Network Operations and Management Symposium. IEEE, 2016, pp. 1193-1196.

[58] S. Uçar, S. Ç. Ergen, and Ö. Özkasap, "Visible light communication in vehicular ad-hoc networks," in Signal Processing and Communication Application Conference (SIU). IEEE, 2016, pp. 881-884.

[59] C. Sommer, O. K. Tonguz, and F. Dressler, "Traffic information systems: efficient message dissemination via adaptive beaconing," IEEE Communications Magazine, vol. 49, no. 5, pp. 173-179, 2011

[60] Q. Zhang, H. Zheng, J. Lan, J. An, and H. Peng, "An Autonomous Information Collection and Dissemination Model for Large-Scale Urban Road Networks," IEEE Transactions on Intelligent Transportation Systems, vol. 17, no. 4, pp. 1085-1095, 2016.

[61] R. Hussain, S. Kim, and H. Oh, "Traffic information dissemination system: Extending cooperative awareness among smart vehicles with only single-hop beacons in VANET," Wireless Personal Communications, vol. 88, no. 2, pp. 151-172, 2016.

[62] A. V. Vasilakos, Z. Li, G. Simon, and W. You, "Information centric network: Research challenges and opportunities," Journal of Network and Computer Applications, vol. 52, pp. 1-10, 2015.

[63] L. Zhang, D. Estrin, J. Burke, V. Jacobson, J. D. Thornton, D. K. Smetters, B. Zhang, G. Tsudik, D. Massey, C. Papadopoulos et al., "Named data networking (ndn) project," Relatório Técnico NDN-0001, Xerox Palo Alto Research Center-PARC, vol. 157, p. 158, 2010.

[64] B. Nour, K. Sharif, F. Li, H. Khelifi, and H. Moungla, "NNCP: A Named Data Network Control Protocol for IoT Applications," in IEEE Conference on Standards for Communications and Networking $(C S C N)$. IEEE, 2018, pp. 1-6.

[65] B. Nour, K. Sharif, F. Li, H. Moungla, A. E. Kamal, and H. Afifi, "NCP: A Near ICN Cache Placement Scheme for IoT-based Traffic Class," in IEEE Global Communications Conference (GLOBECOM). IEEE, 2018, pp. 1-6.

[66] A. Karami and M. Guerrero-Zapata, "An ANFIS-based cache replacement method for mitigating cache pollution attacks in Named Data Networking," Computer Networks, vol. 80, pp. 51-65, 2015

[67] T.-A. Le, N. D. Thai, and P. L. Vo, "The performance of caching strategies in content centric networking," in IEEE International Conference on Information Networking (ICOIN). IEEE, 2017, pp. 628-632.

[68] S. Shailendra, S. Sengottuvelan, H. K. Rath, B. Panigrahi, and A. Simha, "Performance evaluation of caching policies in NDN - an ICN architecture," in IEEE Region 10 Conference (TENCON). IEEE, 2016, pp. 1117-1121.

[69] M. G. Rubinstein, F. B. Abdesslem, M. D. De Amorim, S. R Cavalcanti, R. D. S. Alves, L. H. M. K. Costa, O. C. M. B. Duarte, and M. E. M. Campista, "Measuring the capacity of in-car to in-car vehicular networks," IEEE Communications Magazine, vol. 47, no. 11 , pp. 128-136, 2009.

[70] R. A. Rehman and B.-S. Kim, "LOMCF: Forwarding and Caching in Named Data Networking Based MANETs," IEEE Transactions on Vehicular Technology, vol. 66, no. 10, pp. 9350-9364, 2017.

[71] M. Amadeo, C. Campolo, and A. Molinaro, "Content-centric networking: is that a solution for upcoming vehicular networks?" in Proceedings of the Ninth ACM International Workshop on Vehicular Inter-networking, Systems, and Applications. ACM, 2012, pp. 99-102.

[72] D. Grewe, M. Wagner, and H. Frey, "A Domain-Specific Comparison of Information-Centric Networking Architectures for Connected Vehicles," IEEE Communications Surveys \& Tutorials, vol. 20, no. 3, pp. 2372-2388, 2018

[73] R. W. Coutinho, A. Boukerche, and A. A. Loureiro, "Design Guidelines for Information-Centric Connected and Autonomous Vehicles," IEEE Communications Magazine, vol. 56, no. 10, pp. 85-91, 2018.

[74] R. Hussain, S. H. Bouk, N. Javaid, A. M. Khan, and J. Lee, "Realization of VANET-Based Cloud Services through Named Data Networking," IEEE Communications Magazine, vol. 56, no. 8, pp. 168-175, 2018.

[75] C. Xu, P. Zhang, S. Jia, M. Wang, and G.-M. Muntean, "Video streaming in content-centric mobile networks: Challenges and solutions," IEEE Wireless Communications, vol. 24, no. 5, pp. 157-165, 2017.

[76] H. Khelifi, S. Luo, B. Nour, and H. Moungla, "LQCC: A Link Qualitybased Congestion Control Scheme in Named Data Networks," in 
IEEE Wireless Communications and Networking Conference (WCNC). Marrakech, Morocco: IEEE, Apr 2019.

[77] S. Ostrovskaya, O. Surnin, R. Hussain, S. H. Bouk, J. Lee, N. Mehran, S. H. Ahmed, and A. Benslimane, "Towards Multi-metric Cache Replacement Policies in Vehicular Named Data Networks," in IEEE International Symposium on Personal, Indoor and Mobile Radio Communications (PIMRC). IEEE, 2018, pp. 1-7.

[78] G. Grassi, D. Pesavento, G. Pau, R. Vuyyuru, R. Wakikawa, and L. Zhang, "VANET via named data networking," in IEEE Conference on Computer Communications Workshops (INFOCOM WKSHPS). IEEE, 2014, pp. 410-415.

[79] M. Chen, D. O. Mau, Y. Zhang, T. Taleb, and V. C. Leung, "VENDNET: VEhicular named data NETwork," Vehicular Communications, vol. 1, no. 4, pp. 208-213, 2014.

[80] C. De Castro, C. Raffaelli, and O. Andrisano, "A dynamic hierarchical VANET architecture for Named Data Networking applications," in IEEE International Conference on Communications (ICC). IEEE, 2015, pp. 3659-3665.

[81] A. Bazzi, B. M. Masini, A. Zanella, C. De Castro, C. Raffaelli, and O. Andrisano, "Cellular aided vehicular named data networking," in International Conference on Connected Vehicles and Expo (ICCVE). IEEE, 2014, pp. 747-752.

[82] Y.-H. Ho, C.-H. Lin, S.-J. Sun, and L.-J. Chen, "Emergency application for Vehicle-to-Vehicle Communication using Named Data Networking (eVNDN)," in IEEE International Conference on Ubiquitous and Future Networks (ICUFN). IEEE, 2016, pp. 611-616.

[83] S. H. Ahmed, M. Yaqub, S. Bouk, and D. Kim, "Towards contentcentric traffic ticketing in VANETs: An application perspective," in IEEE International Conference on Ubiquitous and Future Networks (ICUFN). IEEE, 2015, pp. 237-239.

[84] S. H. Ahmed, M. A. Yaqub, S. H. Bouk, and D. Kim, "SmartCop: Enabling Smart Traffic Violations Ticketing in Vehicular Named Data Networks," Mobile Information Systems, vol. 2016, p. 12, 2016.

[85] Z. Yan, S. Zeadally, and Y.-J. Park, "A novel vehicular information network architecture based on named data networking (NDN)," IEEE Internet of Things Journal, vol. 1, no. 6, pp. 525-532, 2014.

[86] M. Amadeo, C. Campolo, and A. Molinaro, "CRoWN: Content-centric networking in vehicular ad hoc networks," IEEE Communications Letters, vol. 16, no. 9, pp. 1380-1383, 2012

[87] — " "Enhancing content-centric networking for vehicular environments," Computer Networks, vol. 57, no. 16, pp. 3222-3234, 2013.

[88] W. Drira and F. Filali, "A Pub/Sub extension to NDN for efficient data collection and dissemination in V2X networks," in IEEE International Symposium on a World of Wireless, Mobile and Multimedia Networks (WoWMoM). IEEE, 2014, pp. 1-7.

[89] S. Kumar, L. Shi, N. Ahmed, S. Gil, D. Katabi, and D. Rus, "Carspeak: a content-centric network for autonomous driving," ACM SIGCOMM Computer Communication Review, vol. 42, no. 4, pp. 259-270, 2012.

[90] Z. Su, Y. Hui, and Q. Yang, "The next generation vehicular networks: a content-centric framework," IEEE Wireless Communications, vol. 24, no. 1, pp. 60-66, 2017.

[91] M. Amadeo, C. Campolo, and A. Molinaro, "Design and analysis of a transport-level solution for content-centric VANETs," in IEEE International Conference on Communications Workshops (ICC). IEEE, 2013, pp. $532-537$.

[92] M. Kuai, X. Hong, and R. R. Flores, "Evaluating interest broadcast in vehicular named data networking," in Research and Educational Experiment Workshop (GREE). IEEE, 2014, pp. 77-78.

[93] C. Contoli, W. Cerroni, F. Callegati, and G. Pau, "Performance of Named Data Networking in urban vehicular communications," in International Conference on Transparent Optical Networks (ICTON). IEEE, 2014, pp. 1-4.

[94] S. H. Bouk, M. A. Yaqub, S. H. Ahmed, and D. Kim, "Evaluating interest/data propagation in vehicular named data networks," in Conference on research in adaptive and convergent systems. ACM, 2015, pp. 256-259.

[95] Y.-T. Yu and M. Gerla, "Information-centric VANETs: A study of content routing design alternatives," in IEEE International Conference on Computing, Networking and Communications (ICNC). IEEE, 2016, pp. $1-5$.

[96] L. Wang, R. Wakikawa, R. Kuntz, R. Vuyyuru, and L. Zhang, "Data naming in vehicle-to-vehicle communications," in IEEE Conference on Computer Communications Workshops (INFOCOM WKSHPS). IEEE, 2012, pp. 328-333.

[97] D. Pesavento, G. Grassi, C. E. Palazzi, and G. Pau, "A naming scheme to represent geographic areas in NDN," in IFIP Wireless Days (WD). IEEE, 2013, pp. 1-3.
[98] W. Drira and F. Filali, "NDN-Q: An NDN query mechanism for efficient V2X data collection," in IEEE International Conference on Sensing, Communication, and Networking Workshops (SECON Workshops). IEEE, 2014, pp. 13-18.

[99] Y.-T. Yu, M. Gerla, and M. Sanadidi, "Scalable VANET content routing using hierarchical bloom filters," Wireless Communications and Mobile Computing, vol. 15, no. 6, pp. 1001-1014, 2015.

[100] X. Liu, M. J. Nicolau, A. Costa, J. Macedo, and A. Santos, “A geographic opportunistic forwarding strategy for vehicular named data networking," in Intelligent Distributed Computing IX. Springer, 2016 , pp. 509-521.

[101] M. Chowdhury, A. Gawande, and L. Wang, "Secure Information Sharing among Autonomous Vehicles in NDN," in IEEE International Conference on Internet-of-Things Design and Implementation (IoTDI). IEEE, 2017, pp. 15-26.

[102] F. M. Modesto and A. Boukerche, "SEVeN: A novel service-based architecture for information-centric vehicular network," Computer Communications, vol. 117, pp. 133 - 146, 2018.

[103] W. Quan, C. Xu, J. Guan, H. Zhang, and L. A. Grieco, "Social cooperation for information-centric multimedia streaming in highway VANETs," in IEEE International Symposium on a World of Wireless, Mobile and Multimedia Networks (WoWMoM). IEEE, 2014, pp. 1-6.

[104] S. H. Bouk, S. H. Ahmed, and D. Kim, "Hierarchical and hash-based naming scheme for vehicular information centric networks," in IEEE International Conference on Connected Vehicles and Expo (ICCVE). IEEE, 2014, pp. 765-766.

[105] — "Hierarchical and hash based naming with Compact Trie name management scheme for Vehicular Content Centric Networks," Computer Communications, vol. 71, pp. 73-83, 2015

[106] M. F. Bari, S. R. Chowdhury, R. Ahmed, R. Boutaba, and B. Mathieu, "A survey of naming and routing in information-centric networks," IEEE Communications Magazine, vol. 50, no. 12, pp. 44-53, 2012.

[107] S. S. Adhatarao, J. Chen, M. Arumaithurai, X. Fu, and K. Ramakrishnan, "Comparison of naming schema in ICN," in IEEE International Symposium on Local and Metropolitan Area Networks (LANMAN). IEEE, 2016, pp. 1-6.

[108] Y. Wang, K. He, H. Dai, W. Meng, J. Jiang, B. Liu, and Y. Chen, "Scalable name lookup in NDN using effective name component encoding," in IEEE International Conference on Distributed Computing Systems (ICDCS). IEEE, 2012, pp. 688-697.

[109] N. L. Van Adrichem and F. A. Kuipers, "Globally accessible names in named data networking," in IEEE Conference on Computer Communications Workshops (INFOCOM WKSHPS). IEEE, 2013, pp. 345-350.

[110] H.-K. Zhang, F. Song, W. Quan, J. Guan, and C. Xu, "Uniform information with a hybrid naming (hn) scheme," Internet Engineering Task Force, Internet-Draft, Oct. 2017, work in Progress. [Online]. Available: https://datatracker.ietf.org/doc/html/draft-zhang-icnrg-hn-07

[111] O. Ascigil, S. Ree, G. Xylomenos, I. Psaras, and G. Pavlou, "A keyword-based ICN-IoT platform," in ACM Conference on Information-Centric Networking (ICN). ACM, 2017, pp. 22-28.

[112] B. Nour, K. Sharif, F. Li, H. Moungla, and Y. Liu, "M2HAV: A Standardized ICN Naming Scheme for Wireless Devices in Internet of Things," in International Conference on Wireless Algorithms, Systems, and Applications (WASA). Springer International Publishing, 2017, pp. 289-301.

[113] S. Arshad, M. A. Azam, S. H. Ahmed, and J. Loo, "Towards Information-Centric Networking (ICN) naming for Internet of Things (IoT): the case of smart campus," in International Conference on Future Networks and Distributed Systems (ICFNDS). ACM, 2017, p. 30.

[114] S. Arshad, B. Shahzaad, M. A. Azam, J. Loo, S. H. Ahmed, and S. Aslam, "Hierarchical and Flat-Based Hybrid Naming Scheme in Content-Centric Networks of Things," IEEE Internet of Things Journal, vol. 5, no. 2, pp. 1070-1080, 2018

[115] L. Wang, A. Afanasyev, R. Kuntz, R. Vuyyuru, R. Wakikawa, and L. Zhang, "Rapid traffic information dissemination using named data," in ACM Workshop on Emerging Name-Oriented Mobile Networking Design-Architecture, Algorithms, and Applications. ACM, 2012, pp. $7-12$.

[116] M. Tarroumi and I. Jabri, "EVNDN: Enhanced vehicular named data networking," in IEEE International Symposium on Networks, Computers and Communications (ISNCC). IEEE, 2017, pp. 1-6.

[117] Y.-T. Yu, Y. Li, X. Ma, W. Shang, M. Sanadidi, and M. Gerla, "Scalable opportunistic VANET content routing with encounter information," in IEEE International Conference on Network Protocols (ICNP). IEEE, 2013, pp. $1-6$

[118] C. Bian, T. Zhao, X. Li, and W. Yan, "Boosting named data networking for efficient packet forwarding in urban vanet scenarios," in IEEE 
International Workshop on Local and Metropolitan Area Networks (LANMAN). IEEE, 2015, pp. 1-6.

[119] — - "Boosting named data networking for data dissemination in urban VANET scenarios," Vehicular Communications, vol. 2, no. 4, pp. 195207, 2015.

[120] G. Grassi, D. Pesavento, G. Pau, L. Zhang, and S. Fdida, "Navigo: Interest forwarding by geolocations in vehicular Named Data Networking," in IEEE International Symposium on World of Wireless, Mobile and Multimedia Networks (WoWMoM). IEEE, 2015, pp. 1-10.

[121] S. H. Ahmed, S. H. Bouk, and D. Kim, "RUFS: RobUst forwarder selection in vehicular content-centric networks," IEEE Communications Letters, vol. 19, no. 9, pp. 1616-1619, 2015.

[122] M. Kuai, X. Hong, and Q. Yu, "Density-Aware Delay-Tolerant Interest Forwarding in Vehicular Named Data Networking," in IEEE Vehicular Technology Conference (VTC-Fall). IEEE, 2016, pp. 1-5.

[123] Z. Lin, M. Kuai, and X. Hong, "Reliable Forwarding Strategy in Vehicular Networks Using NDN," in IEEE Vehicular Technology Conference (VTC-Fall). IEEE, 2016, pp. 1-5.

[124] G. Deng, X. Xie, L. Shi, and R. Li, "Hybrid information forwarding in VANETs through named data networking," in IEEE International Symposium on Personal, Indoor, and Mobile Radio Communications (PIMRC). IEEE, 2015, pp. 1940-1944.

[125] K. Kaur and S. Kad, "Improved Vehicular Information Network Architecture Using Fuzzy Based Named Data Networking (NDN)," International Journal of Scientific \& Technology Research, vol. 4, no. 8, pp. 196-200, 2015.

[126] — "Fuzzy based named data networking (NDN) for vehicular information network," in IEEE International Conference on Next Generation Computing Technologies (NGCT). IEEE, 2016, pp. 382-386.

[127] G. Deng, F. Li, and L. Wang, "Cooperative downloading in VANETsLTE heterogeneous network based on Named Data," in IEEE Conference on Computer Communications Workshops (INFOCOM WKSHPS). IEEE, 2016, pp. 233-238.

[128] F. B. Gonçalves, F. M. França, and C. L. de Amorim, "Interestcentric vehicular ad hoc network," in IEEE International Conference on Wireless and Mobile Computing, Networking and Communications (WiMob), vol. abs/1604.00589. IEEE, 2016, pp. 1-10.

[129] E. Kalogeiton, T. Kolonko, and T. Braun, "A multihop and multipath routing protocol using NDN for VANETs," in IEEE Annual Mediterranean Ad Hoc Networking Workshop (Med-Hoc-Net). IEEE, 2017, pp. $1-8$.

[130] - "A topology-oblivious routing protocol for NDN-VANETs," arXiv preprint arXiv:1711.09870, 2017.

[131] D. Saxena, V. Raychoudhury, and C. Becker, "Implementation and Performance Evaluation of Name-based Forwarding Schemes in VNDN," in International Conference on Distributed Computing and Networking. ACM, 2017, p. 35.

[132] H. Guo, L. Rui, R. Shi, H. Huang, and X. Qiu, "A New ICN routing selecting algorithm based on Link Expiration Time of VANET under the highway environment," in IFIP/IEEE Symposium on Integrated Network and Service Management (IM). IEEE, 2017, pp. 640-643.

[133] K. Husain and A. Awang, "A Receiver-Based Forwarding Scheme to Minimize Multipath Formation in VANET," in Vehicular Ad-Hoc Networks for Smart Cities. Springer, 2017, pp. 15-26.

[134] L. Rui, H. Guo, H. Huang, R. Shi, and X. Qiu, "MUPF: Multiple Unicast Path Forwarding in Content-Centric VANETs," Ad Hoc Networks, vol. 81, pp. 211-225, 2018.

[135] S. H. Ahmed, S. H. Bouk, M. A. Yaqub, D. Kim, and H. Song, "DIFS: Distributed Interest Forwarder Selection in Vehicular Named Data Networks," IEEE Transactions on Intelligent Transportation Systems, vol. 19 , no. 9, pp. 3076-3080, 2017.

[136] H. Maryam, A. Wahid, and M. A. Shah, "Mitigating broadcast storm in interest/data packet forwarding in vehicular content centric networking," in IEEE International Conference on Communication Technologies (ComTech). IEEE, 2017, pp. 162-167.

[137] A. Wahid, M. A. Shah, F. F. Qureshi, H. Maryam, R. Iqbal, and V. Chang, "Big data analytics for mitigating broadcast storm in vehicular content centric networks," Future Generation Computer Systems, vol. 86, pp. $1301-1320,2018$.

[138] R. Soua, E. Kalogeiton, G. Manzo, J. M. Duarte, M. R. Palattella, A. Di Maio, T. Braun, T. Engel, L. A. Villas, and G. A. Rizzo, "SDN coordination for CCN and FC content dissemination in VANETs," in Ad Hoc Networks. Springer, 2017, pp. 221-233.

[139] S. Tiennoy and C. Saivichit, "Using a Distributed Roadside Unit for the Data Dissemination Protocol in VANET with the Named Data Architecture," IEEE Access, vol. 6, pp. 32 612-32 623, 2018.
[140] M. A. Yaqub, S. H. Ahmed, and D. Kim, "BIRD: bio-inspired distributed interest forwarding in vehicular named-data networks," in Annual ACM Symposium on Applied Computing. ACM, 2018, pp. 2078-2083.

[141] G. Arnould, D. Khadraoui, and Z. Habbas, "A self-organizing content centric network model for hybrid vehicular ad-hoc networks," in ACM International Symposium on Design and Analysis of Intelligent Vehicular Networks and Applications. ACM, 2011, pp. 15-22.

[142] M. F. Majeed, S. H. Ahmed, S. Muhammad, and M. N. Dailey, "PDF: Push-based Data Forwarding in Vehicular NDN," in International Conference on Mobile Systems, Applications, and Services Companion. ACM, 2016, pp. 54-54.

[143] M. F. Majeed, S. H. Ahmed, and M. N. Dailey, "Enabling Push-Based Critical Data Forwarding in Vehicular Named Data Networks," IEEE Communications Letters, vol. 21, no. 4, pp. 873-876, 2017.

[144] M. Amadeo, C. Campolo, and A. Molinaro, "Named data networking for priority-based content dissemination in VANETs," in IEEE International Symposium on Personal, Indoor, and Mobile Radio Communications (PIMRC). IEEE, 2016, pp. 1-6.

[145] — "Priority-Based Content Delivery in the Internet of Vehicles through Named Data Networking," Journal of Sensor and Actuator Networks, vol. 5, no. 4, p. 17, 2016.

[146] A. Boukerche, R. W. Coutinho, and X. Yu, "LISIC: A Link StabilityBased Protocol for Vehicular Information-Centric Networks," in IEEE International Conference on Mobile Ad Hoc and Sensor Systems (MASS). IEEE, 2017, pp. 233-240.

[147] P. TalebiFard and V. Leung, "A content centric approach to dissemination of information in vehicular networks," in ACM International Symposium on Design and Analysis of Intelligent Vehicular Networks and Applications. ACM, 2012, pp. 17-24.

[148] P. Talebifard and V. C. Leung, "Towards a content-centric approach to crowd-sensing in vehicular clouds," Journal of Systems Architecture, vol. 59, no. 10, pp. 976-984, 2013.

[149] G. Deng, L. Shi, R. Li, and X. Xie, "Efficient Inter-Vehicle Internet Content Distribution Based on Named Data," in IEEE Vehicular Technology Conference (VTC Fall). IEEE, 2015, pp. 1-5.

[150] R. Li, L. Shi, and S. Li, "A process of high efficiency and low redundancy in content distribution based on Named Data Networking in VANETs," in IEEE International Conference on Computer and Communications (ICCC). IEEE, 2016, pp. 2490-2494.

[151] S. H. Bouk, S. H. Ahmed, Y. Eun, and K.-J. Park, "Maximum Information Coverage in Named Data Vehicular Cyber-Physical Systems," in IEEE International Conference on Communications (ICC). IEEE, 2018, pp. 1-7.

[152] S. H. Ahmed, S. H. Bouk, M. A. Yaqub, D. Kim, and M. Gerla, "CONET: Controlled data packets propagation in vehicular Named Data Networks," in IEEE Consumer Communications \& Networking Conference (CCNC). IEEE, 2016, pp. 620-625.

[153] S. H. Ahmed, S. H. Bouk, M. A. Yaqub, D. Kim, H. Song, and J. Lloret, "CODIE: Controlled data and interest evaluation in vehicular named data networks," IEEE Transactions on Vehicular Technology, vol. 65, no. 6 , pp. 3954-3963, 2016

[154] S. H. Bouk, S. H. Ahmed, M. A. Yaqub, D. Kim, and M. Gerla, "DPEL: Dynamic PIT Entry Lifetime in Vehicular Named Data Networks," IEEE Communications Letters, vol. 20, no. 2, pp. 336-339, 2016.

[155] S. Boussoufa-Lahlah, F. Semchedine, and L. Bouallouche-Medjkoune, "Geographic routing protocols for Vehicular Ad hoc NETworks (VANETs): A survey," Vehicular Communications, vol. 11, pp. $20-$ 31, 2018.

[156] I. Yaqoob, I. Ahmad, E. Ahmed, A. Gani, M. Imran, and N. Guizani, "Overcoming the key challenges to establishing vehicular communication: Is SDN the answer?" IEEE Communications Magazine, vol. 55, no. 7, pp. 128-134, 2017

[157] B. Nour, K. Sharif, F. Li, and H. Moungla, "A Distributed ICN-Based IoT Network Architecture: An Ambient Assisted Living Application Case Study," in IEEE Global Communications Conference (GLOBECOM). IEEE, 2017, pp. 1-6.

[158] M. A. Yaqub, S. H. Ahmed, and D. Kim, "Asking neighbors a favor: Cooperative video retrieval using cellular networks in VANETs," Vehicular Communications, vol. 12, pp. 39 - 49, 2018.

[159] G. Deng, L. Wang, F. Li, and R. Li, "Distributed Probabilistic Caching strategy in VANETs through Named Data Networking," in IEEE Conference on Computer Communications Workshops (INFOCOM WKSHPS). IEEE, 2016, pp. 314-319.

[160] G. Mauri, M. Gerla, F. Bruno, M. Cesana, and G. Verticale, "Optimal Content Prefetching in NDN Vehicle-to-Infrastructure Scenario," IEEE 
Transactions on Vehicular Technology, vol. 66, no. 3, pp. 2513-2525, 2017.

[161] L. C. Liu, D. Xie, S. Wang, and Z. Zhang, "CCN-based cooperative caching in VANET," in IEEE International Conference on Connected Vehicles and Expo (ICCVE). IEEE, 2015, pp. 198-203.

[162] W. Zhao, Y. Qin, D. Gao, C. H. Foh, and H.-C. Chao, "An Efficient Cache Strategy in Information Centric Networking Vehicle-to-Vehicle Scenario," IEEE Access, vol. 5, pp. 12 657-12667, 2017.

[163] D. Grewe, M. Wagner, and H. Frey, "PeRCeIVE: Proactive caching in ICN-based VANETs," in IEEE Vehicular Networking Conference $(V N C)$. IEEE, 2016, pp. 1-8.

[164] H. Khelifi, S. Luo, B. Nour, A. Sellami, H. Moungla, and F. NaïtAbdesselam, "An Optimized Proactive Caching Scheme based on Mobility Prediction for Vehicular Networks," in IEEE Global Communications Conference (GLOBECOM). IEEE, 2018, pp. 1-6.

[165] I. Abdullahi, S. Arif, and S. Hassan, "Survey on caching approaches in Information Centric Networking," Journal of Network and Computer Applications, vol. 56, pp. 48-59, 2015.

[166] F. M. Modesto and A. Boukerche, "An analysis of caching in information-centric vehicular networks," in IEEE International Conference on Communications (ICC). IEEE, 2017, pp. 1-6.

[167] J. Dai, Z. Hu, B. Li, J. Liu, and B. Li, "Collaborative hierarchical caching with dynamic request routing for massive content distribution," in IEEE International Conference on Computer (INFOCOM). IEEE, 2012, pp. 2444-2452.

[168] M. F. Majeed, S. H. Ahmed, S. Muhammad, H. Song, and D. B. Rawat, "Multimedia streaming in information-centric networking: A survey and future perspectives," Computer Networks, vol. 125, pp. 103-121, 2017.

[169] C. E. Perkins, "Mobile networking through mobile IP," IEEE Internet Computing, vol. 2, no. 1, pp. 58-69, 1998.

[170] R. Moskowitz and P. Nikander, "Host Identity Protocol (HIP) Architecture," RFC 4423, May 2006.

[171] G. Tyson, N. Sastry, R. Cuevas, I. Rimac, and A. Mauthe, "A survey of mobility in information-centric networks," Communications of the $A C M$, vol. 56, no. 12, pp. 90-98, 2013.

[172] G. Tyson, N. Sastry, I. Rimac, R. Cuevas, and A. Mauthe, "A survey of mobility in information-centric networks: Challenges and research directions," in ACM Workshop on Emerging Name-Oriented Mobile Networking Design-Architecture, Algorithms, and Applications. ACM, 2012, pp. 1-6.

[173] W. Shang, Y. Yu, R. Droms, and L. Zhang, "Challenges in IoT networking via TCP/IP architecture," Technical Report NDN-0038. NDN Project, 2016.

[174] Y. Zhang, A. Afanasyev, J. Burke, and L. Zhang, "A survey of mobility support in named data networking," in IEEE Conference on Computer Communications Workshops. IEEE, 2016, pp. 83-88.

[175] L. Wang, O. Waltari, and J. Kangasharju, "Mobiccn: Mobility support with greedy routing in content-centric networks," in IEEE Global Communications Conference (GLOBECOM). IEEE, 2013, pp. 20692075.

[176] M. Wang, C. Xu, S. Jia, J. Guan, and L. A. Grieco, "Preferenceaware fast interest forwarding for video streaming in informationcentric VANETs," in IEEE International Conference on Communications (ICC). IEEE, 2017, pp. 1-7.

[177] J. M. Duarte, T. Braun, and L. A. Villas, "Receiver mobility in vehicular named data networking," in Workshop on Mobility in the Evolving Internet Architecture. ACM, 2017, pp. 43-48.

[178] — "Addressing the Effects of Low Vehicle Density in Highly Mobile Vehicular Named-Data Networks," in ACM Symposium on Development and Analysis of Intelligent Vehicular Networks and Applications. ACM, 2017, pp. 117-124.

[179] _ _ "Source Mobility in Vehicular Named-Data Networking: An Overview," in Ad Hoc Networks. Springer, 2018, pp. 83-93.

[180] — "MobiVNDN: A Distributed Framework to Support Mobility in Vehicular Named-Data Networking," Ad hoc Networks, pp. 77-90, 2018.

[181] M. Wang, J. Wu, G. Li, J. Li, Q. Li, and S. Wang, "Toward Mobility Support for Information-Centric IoV in Smart City Using Fog Computing," in IEEE International Conference on Smart Energy Grid Engineering. IEEE, 2017, pp. 357-361.

[182] R. Tourani, S. Misra, T. Mick, and G. Panwar, "Security, privacy, and access control in information-centric networking: A survey," IEEE Communications Surveys \& Tutorials, vol. 20, no. 1, pp. 566-600, 2017.
[183] H. Khelifi, S. Luo, B. Nour, and S. C. Shah, "Security \& Privacy Issues in Vehicular Named Data Networks: An Overview," Mobile Information Systems, vol. 2018, p. 11, 2018.

[184] Z. Rezaeifar, J. Wang, and H. Oh, "A trust-based method for mitigating cache poisoning in Name Data Networking," Journal of Network and Computer Applications, vol. 104, pp. 117-132, 2018.

[185] J. Wang, R. Wakikawa, and L. Zhang, "DMND: Collecting data from mobiles using named data," in IEEE Vehicular networking conference $(V N C)$. IEEE, 2010, pp. 49-56.

[186] H. Khelifi, S. Luo, B. Nour, H. Moungla, and S. H. Ahmed, "Reputation-based Blockchain for Secure NDN Caching in Vehicular Networks," in IEEE Conference on Standards for Communications and Networking (CSCN). IEEE, 2018, pp. 1-6.

[187] M. Chowdhury, A. Gawande, and L. Wang, "Anonymous authentication and pseudonym-renewal for VANET in NDN," in ACM Conference on Information-Centric Networking. ACM, 2017, pp. 222-223.

[188] M. Mosko, I. Solis, and C. A. Wood, "CCNx Semantics," Internet Engineering Task Force, Internet-Draft, Jun. 2018, work in Progress. [Online]. Available: https://datatracker.ietf.org/doc/html/ draft-irtf-icnrg-ccnxsemantics-09

[189] —, "CCNx Messages in TLV Format," Internet Engineering Task Force, Internet-Draft, Jul. 2018, work in Progress. [Online]. Available: https://datatracker.ietf.org/doc/html/draft-irtf-icnrg-ccnxmessages-08

[190] "FD.io - The Fast Data Project," [Online] https://fd.io/, Accessed: 201809-17.

[191] “CCN-lite Project," [Online] http://ccn-lite.net/, Accessed: 2018-09-17.

[192] "ICN2020: Horizon 2020 Project," [Online] http://www.icn2020.org/, Accessed: 2018-09-17.

[193] "Design Choices and Differences for NDN and CCNx 1.0 Implementations of Information-Centric Networking," Internet Engineering Task Force, Internet-Draft, Jul. 2017, work in Progress. [Online]. Available: https://icnrg.github.io/draft-ienrg-harmonization/ draft-icnrg-harmonization-00.html

[194] A. Afanasyev, I. Moiseenko, and L. Zhang, "ndnSIM: NDN simulator for NS-3," Tech. Rep. NDN-0005, pp. 1-7, 2012.

[195] R. Chiocchetti, D. Rossi, and G. Rossini, "ccnsim: An highly scalable ccn simulator," in IEEE International Conference on Communications (ICC). IEEE, 2013, pp. 2309-2314.

[196] T. Issariyakul and E. Hossain, Introduction to Network Simulator NS2, 1st ed. Springer Publishing Company, Incorporated, 2010.

[197] L. Saino, I. Psaras, and G. Pavlou, "Icarus: a caching simulator for information centric networking (ICN)," in International ICST conference on Simulation Tools and Techniques. ICST (Institute for Computer Sciences, Social-Informatics and Telecommunications Engineering), 2014, pp. 66-75.

[198] A. Vargas, "OMNeT++ Discrete Event Simulation System User Manual," 2005.

[199] "QualNet," [Online] http://www.qualnet.com/, Accessed: 2018-09-17.

[200] H. Khelifi, S. Luo, B. Nour, A. Sellami, H. Moungla, S. H. Ahmed, and M. Guizani, "Bringing Deep Learning at The Edge of InformationCentric Internet of Things," IEEE Communications Letters, vol. 23, no. 1 , pp. 52-55, 2019.

\section{BIOGRAPHIES}

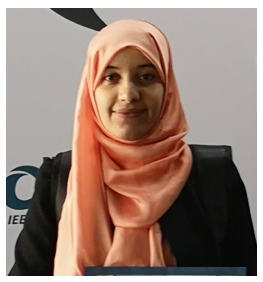

Hakima Khelifi [GS'17] is currently pursuing a $\mathrm{Ph} . \mathrm{D}$. degree in Information and Communication Engineering from Beijing Institute of Technology, Beijing, China. She received a B.Sc. degree in Computer Science (2013) from Constantine 2 University, Constantine, Algeria, and a M.Sc. degree (2015) from Mohamed El Bachir El Ibrahimi University, Bordj Bou-Arreridj, Algeria. She received the Best Paper Award of the IEEE GLOBECOM in 2018, and the Excellent Student Award at the Beijing Institute of Technology in 2017-18. Her current research interest includes Next-Generation Networking and Internet, NamedData Networking, and Vehicular ad hoc Networks. She is a Graduate Student Member of IEEE. 


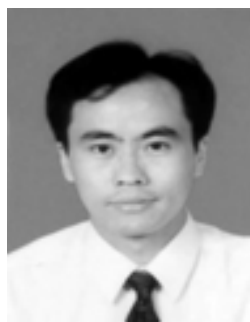

Senlin Luo received the B.E. and M.E. degrees from the college of Electrical and Electronic Engineering, Harbin University of Science and Technology, Harbin, China, in 1992 and 1995 respectively, and the Ph.D. degree from the School of Information and Electronics, Beijing Institute of Technology, Beijing, China, in 1998. He is currently a Deputy Director, Laboratory Director, and Professor of Information System and Security Countermeasures Experimental Center, Beijing Institute of Technology. His current research interests include Machine Learning, Medical Data Mining, and Information Security.

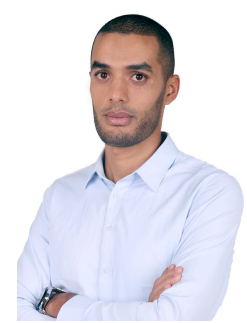

Boubakr Nour [GS'17] is pursuing his Ph.D. in Computer Science \& Technology at Beijing Institute of Technology, Beijing, China. Previously, he received both M.Sc. and B.Sc. degrees with distinction in Computer Science from Djillali Liabes University, Sidi Bel Abbes, Algeria, in 2016, and 2014 respectively. Also, he has been a visiting researcher at Paris Descartes University, Paris, France, in 2016. He received the Best Paper Award of the IEEE GLOBECOM 2018, and won the Excellent Student Award at the Beijing Institute of Technology in 2016-17 and 2017-18. His research interests include Next-Generation Networking and Internet, Information-Centric Networking, Mobile/Edge Computing, and Internet of Things.

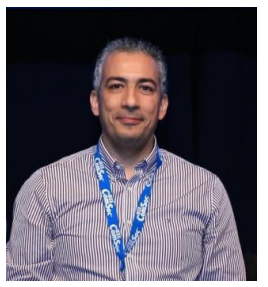

Hassine Moungla $[\mathbf{M}]$ is Associate Professor at the University of Paris Descartes and a member of the Paris Descartes Computer Science Laboratory (LIPADE) since October 2008. He was a researcher at INRIA until 2008 and research fellow at CNRS-LIPN laboratory of the Paris Nord University until 2007. His research interests lie in the field of Wireless Area Body Networking (WBAN) for medical and health applications, Wireless Sensor Networking, QoS in WSN, Middleware for 5G Mobile and Sensor Networks. He participated and still participates to several national and international research projects. He is on the technical program committee of different ACM and IEEE conferences, including Globecom, ICC, WCNC, PIMRC, IWCMC and chaired some of their sessions. He is also reviewer on a regular basis for major international journals. Dr. Moungla is a member of IEEE and IEEE Communication Society.

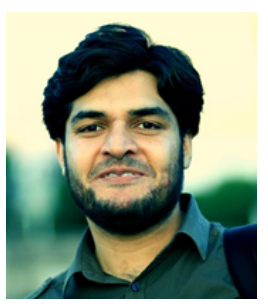

Yasir Faheem received the Ph.D. degree in Computer Science from the Université Paris Nord, France, in 2012, the MS degree in Networks and Distributed Systems from the Université de NiceSophia Antipolis, France, in 2008, and the BS degree in Computer Science from NUCES-FAST, Pakistan, in 2006. He is an Assistant Professor at the Department of Computer Science, COMSATS University Islamabad, Islamabad campus, Pakistan. His current research interests are in the Internet of Things, cloud computing, and wireless ad hoc networks (sensor networks, cognitive radio networks, opportunistic networks). Moreover, he is interested in the applications of the algorithmic game theory to various domains. He has served on the technical program committee of the IEEE ICC, IEEE GLOBECOM and various other conferences. Moreover, he regularly serves as a reviewer for the IEEE Communications Magazine and the Elsevier Journal of Network and Computer Applications. He is also an editorial board member for the IEEE Internet Policy and the IEEE Future Directions newsletters.

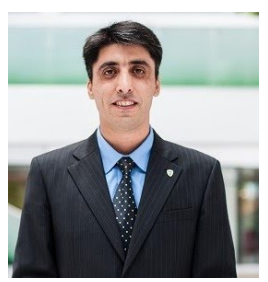

Rasheed Hussain [M] received his B.S. Engineering degree in Computer Software Engineering from University of Engineering and Technology, Peshawar, Pakistan in 2007, MS and PhD degrees in Computer Science and Engineering from Hanyang University, South Korea in 2010 and 2015, respectively. He worked as a Postdoctoral Fellow at Hanyang University, South Korea from March 2015 to August 2015. He also worked as a guest researcher and consultant at University of Amsterdam (UvA) from September 2015 till May 2016. He worked as Assistant Professor at Innopolis University, Innopolis Russia from June 2016 till December 2018. Currently he is an Associate Professor and Head of the MS program in Secure System and Network Engineering (SNE) at Innopolis University, Russia. He is also the Head of Network, Cyber, and Information Security (NCIS) Lab at Innopolis University. He serves as editorial board member for various journals including IEEE Access, IEEE Internet Initiative, Internet Technology Letters, Wiley, and serves as reviewer for most of the IEEE transactions, Springer and Elsevier Journals. He also serves as technical program committee member of various conferences such as IEEE VTC, IEEE VNC, IEEE Globecom, IEEE ICCVE, and so on. He is a certified trainer for Instructional Skills Workshop (ISW). Furthermore, he is also ACM Distinguished Speaker. His research interests include Information Security and Privacy and particularly security and privacy issues in Vehicular Ad Hoc NETworks (VANETs), vehicular clouds, and vehicular social networking, applied cryptography, Internet of Things, Content-Centric Networking (CCN), cloud computing, and blockchain. Currently he is working on carpooling and blockchain technologies for resource-constrained environments.

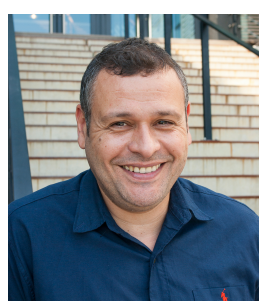

Adlen Ksentini [SM] is a COMSOC distinguished lecturer. He obtained his Ph.D. degree in computer science from the University of Cergy-Pontoise in 2005 , with a dissertation on QoS provisioning in IEEE 802.11-based networks. From 2006 to 2016, he worked at the University of Rennes 1 as an assistant professor. During this period, he was a member of the Dionysos Team with INRIA, Rennes. Since March 2016, he has been working as an assistant professor in the Communication Systems Department of EURECOM. He has been involved in several national and European projects on QoS and QoE support in future wireless, network virtualization, cloud networking, mobile networks, and more recently on Network Slicing and 5G in the context of H2020 projects 5G!Pagoda and 5GTransformer. He has co-authored over 100 technical journal and international conference papers. He received the best paper award from IEEE IWCMC 2016, IEEE ICC 2012, and ACM MSWiM 2005. He has been awarded the 2017 IEEE Comsoc Fred W. Ellersick (best IEEE communications Magazine's paper). Adlen Ksentini has given several tutorials in IEEE international conferences, IEEE Globecom 2015, IEEEE CCNC 2017, IEEE ICC 2017, IEEE/IFIP IM 2017. Adlen Ksentini has been acting as TPC Symposium Chair for IEEE ICC 2016/2017, IEEE GLOBECOM 2017, IEEE Cloudnet 2017 and IEEE 5G Forum 2018. He has been acting as Guest Editor for IEEE Journal of Selected Area on Communication (JSAC) Series on Network Softwerization, IEEE Wireless Communications, IEEE Communications Magazine, and two issues of ComSoc MMTC Letters. He has been on the Technical Program Committees of major IEEE ComSoc, ICC/GLOBECOM, ICME, WCNC, and PIMRC conferences. He is currently the Director of IEEE ComSoc EMEA region and Vice-Chair of the IEEE ComSoc Technical Committee on Software (TCS). 